\title{
REPRESENTATIONS OF COMPLEX SEMI-SIMPLE LIE GROUPS AND LIE ALGEBRAS
}

\author{
APOORVA KHARE ${ }^{\dagger}$
}

\begin{abstract}
This article is an exposition of the 1967 paper by Parthasarathy, Ranga Rao, and Varadarajan, on irreducible admissible Harish-Chandra modules over complex semisimple Lie groups and Lie algebras. It was written in Winter 2012 to be part of a special collection organized to mark 10 years and 25 volumes of the series Texts and Readings in Mathematics (TRIM). Each article in this collection is intended to give nonspecialists in its field, an appreciation for the impact and contributions of the paper being surveyed. Thus, the author has kept the prerequisites for this article down to a basic course on complex semisimple Lie algebras.

While it arose out of the grand program of Harish-Chandra on admissible representations of semisimple Lie groups, the work by Parthasarathy et al also provided several new insights on highest weight modules and related areas, and these results have been the subject of extensive research over the last four decades. Thus, we also discuss its results and follow-up works on the classification of irreducible Harish-Chandra modules; on the PRV conjecture and tensor product multiplicities; and on PRV determinants for (quantized) affine and semisimple Lie algebras.
\end{abstract}

\section{Contents}

1. Notation and preliminaries

2. Harish-Chandra modules

3. Tensor products, minimal types, and the (K)PRV conjecture

4. Irreducible Banach space representations

5. The rings $\mathcal{R}_{\nu, \Pi^{\prime}}$ and the PRV determinants

6. Representations of class zero

7. Conclusion: The classification of irreducible Harish-Chandra modules References

Lie groups and Lie algebras occupy a prominent and central place in mathematics, connecting differential geometry, representation theory, algebraic geometry, number theory, and theoretical physics. In some sense, the heart of (classical) representation theory is in the study of the semisimple Lie groups. Their study is simultaneously simple in its beauty, as well as complex in its richness. From Killing, Cartan, and Weyl, to Dynkin, Harish-Chandra, Bruhat, Kostant, and Serre, many mathematicians in the twentieth century have worked on building up the theory of semisimple Lie algebras and their universal enveloping algebras. Books by Borel, Bourbaki, Bump, Chevalley, Humphreys, Jacobson, Varadarajan, Vogan, and others form the texts for (introductory) graduate courses on the subject.

Date: September 7, 2012.

2010 Mathematics Subject Classification. Primary: 22E46; Secondary: 17B20.

$\dagger$ Departments of Mathematics and Statistics, Stanford University, Stanford, CA - 94305, USA.

Email: khare@stanford.edu.

This work was supported in part by DARPA Grant \# YFA N66001-11-1-4131. 
The purpose of this article is to provide an exposition of the famous 1967 paper [PRV2] by Parthasarathy, Ranga Rao, and Varadarajan on a class of irreducible Banach space representations of a complex semisimple Lie group. This paper was written in a period containing some of the other classic works in the subject: Harish-Chandra's pioneering work on the principal series representations, and his results on the annihilators of simple modules and central characters; Kostant's work on harmonic polynomials and on his character formula; and papers about Steinberg's formula and Verma modules, to name a few.

In this article, we attempt to explain some of the key ideas and main results of [PRV2]. Given the wide variety of new concepts proposed, as well as its impact on subsequent research in the field, the paper ranks alongside these other works mentioned above.

0.1. The basic motivation for the paper PRV2 arose out of the important works Har2, Har3 of Harish-Chandra, in which he constructed a large family of infinite-dimensional irreducible representations of a real semisimple Lie group $G$. Harish-Chandra generalized the constructions by Gelfand and Naimark in the case when $G$ is complex semisimple, and his work is regarded today as a cornerstone in the field. For instance, he showed how irreducible representations are subquotients of the principal series representations.

In their work, which followed a few years after [Har2, Har3], Parthasarathy et al returned to the simpler setting of complex semisimple Lie groups, where they were able to use HarishChandra's results to obtain a deeper understanding of the structure of Harish-Chandra's Banach space representations of $G$. Their paper develops a beautiful theory of such representations, each of which decomposes into finite-dimensional modules when restricted to the maximal compact subgroup of $G$. The authors go on to develop the theory of minimal types, and refine Harish-Chandra's methods (in the complex case) for classifying such irreducible Banach space representations.

0.2. Although this is the primary motivation for [PRV2], the paper develops and proves many other results that have since influenced and inspired a large body of research in the field. We mention a few of these here (and elaborate upon them in future sections). First, the authors provided a multiplicity formula for the classical "tensor product decomposition" problem: given two simple finite-dimensional modules over a complex semisimple Lie algebra, can one write down the decomposition of their tensor product? Combinatorial results due to Kostant, Sternberg, and Brauer were known at the time; however, they required double summations over the Weyl group, computing the Kostant partition function, and cancelling terms in the summation, which made them increasingly harder to implement.

In [PRV2], the authors proposed a formula which was somewhat simpler, directly involving the tensor factors in question. This formula has since been widely used in the literature (as we point out in this article), including in the setting of quantum affine algebras and symmetrizable Kac-Moody algebras, as well as current algebras and other semidirect product Lie algebras.

0.3. Next, a byproduct of this "PRV Theorem" (or formula) was that every such tensor product contains a unique "largest" summand (the "Cartan component"), and a unique "smallest" summand (the "PRV component", or "minimal type"). The former was well-known to be the sum of the two highest weights in question, but the latter was new. Subsequently, the authors and Kostant conjectured the existence of other components, the so-called "generalized PRV components". These are simple modules that occur as direct summands of the tensor product, and their (dominant integral) highest weights are Weyl group-linear combinations of the highest weights of the two tensor factors.

This "PRV Conjecture" has since been proved using multiple techniques in the semisimple as well as Kac-Moody settings. Moreover, it has inspired subsequent research that has led to many 
contributions in understanding the original problem of computing tensor product multiplicities. Once again, we will discuss these facts in detail below. Throughout this article, we will discuss the results in $[\mathrm{PRV} 2]$ in the special case of $\mathfrak{s l}_{2}(\mathbb{C})$, in order to provide a working example - one which we hope will give the reader a greater feel for the results being stated.

0.4. We end this introduction with one last application. In PRV2], using Kostant's "separation of variables" theorem, the authors defined a set of matrices indexed by pairs of dominant integral weights, whose entries are polynomials on the Cartan subalgebra. The determinants of these matrices yield information about the annihilators of Verma modules, and of their simple quotients. (This is related to the Shapovalov form.)

These "PRV determinants" have since been widely studied, not just in the semisimple case, but in the quantum (affine) and the super-reductive settings as well. In these settings, PRV determinants can be used to determine whether or not the annihilators of Verma modules are generated by their intersection with the center.

\section{Notation AND PRELIMINARIES}

We assume for the purposes of this article that the reader is familiar with basic results concerning the structure of complex semisimple Lie algebras; see [Hu1], for instance. We now set some basic notation, which also serves as a quick summary of the theory. Given a complex semisimple Lie algebra $\mathfrak{g}$, fix a Cartan subalgebra $\mathfrak{h} \subset \mathfrak{g}$ (which is abelian and self-normalizing in $\mathfrak{g}$ ). Then $\mathfrak{g}$ has a direct sum decomposition: $\mathfrak{g}=\mathfrak{h} \oplus \bigoplus_{\alpha \in R} \mathfrak{g}_{\alpha}$, where $R$ is the set of roots and $\mathfrak{g}_{\alpha}$ is the one-dimensional root space for each $\alpha \in R \subset \mathfrak{h}^{*}$. Here, an element $\lambda \in \mathfrak{h}^{*}$ is called a weight (in PRV2 it is called a "rank"), and if $M$ is an $\mathfrak{h}$-module, then its $\lambda$-weight space is defined to be:

$$
M_{\lambda}:=\{m \in M: h \cdot m=\lambda(h) m \forall h \in \mathfrak{h}\} .
$$

The weights of $M$, denoted $\operatorname{wt}(M)$, are those $\lambda \in \mathfrak{h}^{*}$ for which $M_{\lambda} \neq 0$. $M$ is a weight module if $M=\bigoplus_{\lambda \in \mathfrak{h}^{*}} M_{\lambda}$. For instance, $\mathfrak{g}$ is a $\mathfrak{g}$-module under the adjoint action, and a weight $\mathfrak{h}$-module. The nonzero weights of $\mathfrak{g}$ are precisely the roots: $\operatorname{wt}(\mathfrak{g})=R \coprod\{0\}$.

A simple example to keep in mind is $\mathfrak{g}=\mathfrak{s l}_{2}(\mathbb{C})$. This has a basis $\{e, f, h\}$ with defining relations:

$$
[h, e]=2 e, \quad[h, f]=-2 f, \quad[e, f]=h .
$$

Here, $\mathfrak{h}=\mathbb{C} \cdot h$ and $R=\{ \pm \alpha\}$, where $\alpha(h)=2$. Thus, $\mathfrak{g}_{\alpha}=\mathbb{C} \cdot e, \mathfrak{g}_{-\alpha}=\mathbb{C} \cdot f$.

1.1. Weights and lattices. Now let $W$ be the associated Weyl group and $(-,-)$ the Killing form for $\mathfrak{g}$. Then $(-,-)$ induces an isomorphism $: \mathfrak{h} \rightarrow \mathfrak{h}^{*}$. Fix a positive system $R^{+} \subset R$ of roots - or equivalently, the subset $\Pi=\left\{\alpha_{i}: i \in I\right\}$ of simple roots indexed by a set $I$. Then $\Pi$ is a basis of $\mathfrak{h}^{*}$, and $R=R^{+} \coprod R^{-}$, where $R^{+}=-R^{-}=R \cap \mathbb{Z}_{\geq 0} \Pi \subset \mathfrak{h}^{*}$. For each $i \in I$, suppose $h_{i}^{\prime} \longleftrightarrow \alpha_{i}$ via the Killing form; now define the co-roots to be $h_{i}:=\left(2 / \alpha_{i}\left(h_{i}^{\prime}\right)\right) \cdot h_{i}^{\prime} \in \mathfrak{h}$.

Next, choose Chevalley generators $e_{i} \in \mathfrak{g}_{\alpha_{i}}$ and $f_{i} \in \mathfrak{g}_{-\alpha_{i}}$ that generate (as above) a copy of $\mathfrak{s l}_{2}$ together with $h_{i}$. Then the $e_{i}$ and $f_{i}$ generate nilpotent subalgebras $\mathfrak{n}^{ \pm}$of $\mathfrak{g}$, and the corresponding Borel (maximal solvable) subalgebras are: $\mathfrak{b}^{ \pm}:=\mathfrak{h} \oplus \mathfrak{n}^{ \pm}$.

We now come to distinguished lattices inside the set of weights. A weight $\lambda \in \mathfrak{h}^{*}$ is said to be dominant if $\lambda\left(h_{i}\right) \geq 0$ for all $i \in I$, and integral if $\lambda\left(h_{i}\right) \in \mathbb{Z}$. The set of integral weights is a lattice $\Lambda \subset \mathfrak{h}^{*}$, whose $\mathbb{Z}$-basis is the set of fundamental weights $\left\{\varpi_{i}: i \in I\right\}$. They are defined by: $\varpi_{i}\left(h_{j}\right):=\delta_{i j}$. Let $\Lambda^{+}$denote the set of dominant integral weights - which are simply $\mathbb{Z}_{\geq 0}$-linear combinations of the $\varpi_{i}$. Then $\Lambda^{+}$is also (in bijection with) the set of dominant characters of a maximal torus $T$ of $G$ (where $G$ is a complex connected Lie group such that $\mathfrak{g}=\operatorname{Lie}(G)$ ). 
The weight lattice $\Lambda$ also contains the root lattice $\mathbb{Z} \Pi$ with $\mathbb{Z}$-basis $\Pi$. The group $W$ acts on $\mathfrak{h}^{*}$ and preserves either lattice. It is generated by the simple reflections $\left\{s_{i}: i \in I\right\}$ which act via: $s_{i}(\lambda):=\lambda-2 \lambda\left(h_{i}\right) \alpha_{i}$. The reflections $s_{i}$ satisfy the Coxeter relations according to the Dynkin diagram of $\mathfrak{g}$, and $W$ is a finite group with a well-defined length-function $\ell: W \rightarrow \mathbb{Z}_{\geq 0}$, the associated Bruhat order, and a unique longest element $w_{\circ}=w_{\circ}^{-1}$.

For example, for $\mathfrak{g}=\mathfrak{s l}_{2}(\mathbb{C}), \Pi=R^{+}=\{\alpha\}$, where $\alpha(h)=1$. The associated fundamental weight is $\varpi=\frac{1}{2} \alpha$, so that $\mathbb{Z} \Pi=2 \Lambda$ and $\Lambda^{+}=\mathbb{Z}_{\geq 0} \varpi$. Moreover, $W=S_{2}=\left\{1, s=w_{\circ}\right\}$, where $w_{\circ} \lambda=-\lambda$ for all weights $\lambda \in \mathfrak{h}^{*}=\mathbb{C} \varpi=\mathbb{C} \alpha$.

1.2. Finite-dimensional representations. A representation or module of a group $G$ is simply a group homomorphism $\varpi: G \rightarrow G L(V)$ for some (real or complex) vector space $V$. Similarly, a $\mathfrak{g}$-module $V$ is a Lie algebra homomorphism $\varpi: \mathfrak{g} \rightarrow \operatorname{End}(V)=\mathfrak{g l}(V)$. We say that $\varpi$ is irreducible if $V$ has no nonzero proper submodule; completely reducible or semisimple if $V$ is a direct sum of irreducible submodules; and finite-dimensional if $\operatorname{dim} V<\infty$.

When $\mathfrak{g}$ is semisimple, the irreducible finite-dimensional representations are all weight modules for $\mathfrak{h}$, and parametrized by $\Lambda^{+}$. Here is a quick construction: suppose $\mathfrak{U} \mathfrak{g}$ is the universal enveloping algebra of $\mathfrak{g}$, and for any $\lambda \in \mathfrak{h}^{*}$, let $I_{\lambda}$ be the left $\mathfrak{U} \mathfrak{g}$-ideal generated by $\operatorname{ker} \lambda \subset \mathfrak{h}$ and $\left\{e_{i}: i \in I\right\}$. The Verma module $M(\lambda)$ is defined to be the quotient $\mathfrak{U} \mathfrak{g} / I_{\lambda} . M(\lambda)$ is a weight module and $\operatorname{wt}(M)=\lambda-\mathbb{Z}_{\geq 0} \Pi$. Moreover, a cyclic generator of $M(\lambda)$ lies in $M(\lambda)_{\lambda}=\mathbb{C} \cdot \overline{1_{\mathfrak{L} \mathfrak{g}}}$, which is called the "highest weight space".

The modules $M(\lambda)$ were studied by Verma in his thesis and in Ve]; they are of tremendous importance in representation theory - not only for semisimple Lie algebras, but also Kac-Moody and Virasoro algebras, quantum groups, and other algebras with triangular decomposition. Every Verma module $M(\lambda)$ has a largest maximal submodule and hence a unique simple quotient; denote this by $V(\lambda)$. Then $V(\lambda)$ also has the same properties as $M(\lambda)$ (mentioned in the previous paragraph); moreover, the modules $V(\lambda)$ are pairwise non-isomorphic for $\lambda \in \mathfrak{h}^{*}$.

Note that $V(\lambda)$ is finite-dimensional if and only if $\lambda \in \Lambda^{+}$, and these exhaust all finitedimensional simple $\mathfrak{g}$-modules up to isomorphism. The dual space to a $\mathfrak{g}$-module is also a $\mathfrak{g}$ module; for instance, $V(\lambda)^{*} \cong V\left(-w_{\circ} \lambda\right)$ if $\lambda \in \Lambda^{+}$. Moreover, every finite-dimensional $\mathfrak{g}$-module is semisimple; in other words, every indecomposable finite-dimensional $\mathfrak{g}$-module is irreducible.

For example, if $\mathfrak{g}=\mathfrak{s l}_{2}$, then for every $0 \leq n \in \mathbb{Z}$, there exists a unique irreducible $\mathfrak{s l}_{2}$-module $V(n) \cong V(n)^{*}$ of dimension $n+1$. (Note that we are abusing notation by using $V(n)$ to refer to $V(n \varpi)$.) $V(n)$ contains a vector $v_{n}$ of weight $n$ (a "highest weight vector"), and a basis $v_{n-2 i}:=\left(f^{i} / i !\right) v_{n}$ of weight vectors, for $0 \leq i<\operatorname{dim} V(n)$. One checks that for all $i$,

$$
h \cdot v_{n-2 i}:=(n-2 i) v_{n-2 i}, \quad e \cdot v_{n-2 i}=(n-i+1) v_{n-2 i+2}, \quad f \cdot v_{n-2 i}=(i+1) v_{n-2 i-2},
$$

where we set $v_{n+2}=v_{-n-2}=0$. A concrete example of $V(n)$ is provided by the space of homogeneous polynomials in $X, Y$ of total degree $n$. Define

$$
P_{n}:=\operatorname{ker}\left(-n+X \frac{\partial}{\partial X}+Y \frac{\partial}{\partial Y}\right) \subset \mathbb{C}[X, Y] .
$$

Now define $\rho_{n}: \mathfrak{s l}_{2} \rightarrow \operatorname{End}_{\mathbb{C}}\left(P_{n}\right)$ via:

$$
\rho_{n}(e):=X \frac{\partial}{\partial Y}, \quad \rho_{n}(f):=Y \frac{\partial}{\partial X}, \quad \rho_{n}(h):=X \frac{\partial}{\partial X}-Y \frac{\partial}{\partial Y} .
$$

Then $P_{n} \cong V(n)$ as $\mathfrak{s l}_{2}$-modules.

1.3. Central characters. Given an associative algebra $A$, denote its center by $Z(A)$. An important tool in studying Verma modules and finite-dimensional modules over a (complex) semisimple Lie algebra $\mathfrak{g}$ is the center $Z(\mathfrak{U} \mathfrak{g})$. Classical results of Chevalley and Harish-Chandra 
imply that this is a polynomial algebra in $|I|$ (algebraically independent) generators. Moreover, for all $\lambda \in \mathfrak{h}^{*}$, there exists a central character (i.e., an algebra homomorphism) $\chi(\lambda): Z(\mathfrak{U} \mathfrak{g}) \rightarrow \mathbb{C}$, such that ker $\chi(\lambda)$ kills $M(\lambda)$ and hence $V(\lambda)$ for all $\lambda \in \mathfrak{h}^{*}$. In particular, every $z \in Z(\mathfrak{U} \mathfrak{g})$ acts on $V(\lambda)$ by a scalar (for each $\lambda$ ).

The following important results due to Harish-Chandra completely classify and explain better, the set of central characters. (These are also known as infinitesimal characters in the literature.) To state the results, we need some notation: define $\rho:=\frac{1}{2} \sum_{\alpha \in R^{+}} \alpha \in \mathfrak{h}^{*}$; then $\rho=\sum_{i \in I} \varpi_{i} \in$ $\Lambda^{+}$and $w_{\circ} \rho=-\rho$. Now define the twisted action of $W$ on $\mathfrak{h}^{*}$ via:

$$
w * \lambda:=w(\lambda+\rho)-\rho, \quad \forall w \in W, \lambda \in \mathfrak{h}^{*} .
$$

Then $w *$ - induces an algebra automorphism of Sym $\mathfrak{h}=P\left(\mathfrak{h}^{*}\right)$ (the space of complex polynomials on $\left.\mathfrak{h}^{*}\right)$ for all $w \in W$. Moreover, given any $w \in W$, define $\mathfrak{n}_{w}^{ \pm}:=\bigoplus_{\alpha \in R \cap \mathbb{Z}_{\geq 0}(w \Pi)} \mathfrak{g}_{ \pm \alpha}$. Then $\mathfrak{g}=\mathfrak{n}_{w}^{-} \oplus \mathfrak{h} \oplus \mathfrak{n}_{w}^{+}$for all $w \in W$; for example, when $w=1$, this decomposition is precisely $\mathfrak{g}=\mathfrak{n}^{-} \oplus \mathfrak{h} \oplus \mathfrak{n}^{+}$. Hence $(\mathfrak{U} \mathfrak{g})_{0} \subset \mathfrak{U} \mathfrak{h} \oplus \mathfrak{n}_{w}^{-}(\mathfrak{U} \mathfrak{g}) \mathfrak{n}_{w}^{+}$by the Poincaré-Birkhoff-Witt theorem. Define the Harish-Chandra map $\beta^{w \Pi}$ to be the projection : $(\mathfrak{U} \mathfrak{g})_{0} \rightarrow \mathfrak{U} \mathfrak{h}=\operatorname{Sym} \mathfrak{h}$.

Theorem $1.2([\operatorname{Har} 1])$. For all $w \in W, \beta^{w \Pi}$ is a ring homomorphism $:(\mathfrak{U} \mathfrak{g})_{0} \rightarrow$ Sym $\mathfrak{h}$, which restricts to a ring isomorphism $: Z(\mathfrak{U} \mathfrak{g}) \rightarrow(\operatorname{Sym} \mathfrak{h})^{(W, *)}$. Moreover, for all $\lambda \in \mathfrak{h}^{*}, \chi(\lambda)=\lambda \circ \beta^{\Pi}$. (Here, $\lambda$ extends to an algebra map on $\mathrm{Sym} \mathfrak{h}$.) Every character of $Z(\mathfrak{U} \mathfrak{g})$ equals $\chi(\lambda)$ for some $\lambda \in \mathfrak{h}^{*}$. Moreover, $\chi(\lambda)=\chi(\mu) \Leftrightarrow \lambda=w * \mu$ for some $w \in W$.

For instance, when $\mathfrak{g}=\mathfrak{s l}_{2}(\mathbb{C}),|I|=1$ and

$$
Z\left(\mathfrak{U}\left(\mathfrak{s l}_{2}(\mathbb{C})\right)\right)=\mathbb{C}[\Delta], \quad \Delta=4 f e+h^{2}+2 h, \quad \beta^{\Pi}(\Delta)=h^{2}+2 h .
$$

Then for all $z \in \mathbb{C}$, the Casimir element $\Delta$ acts on $V(z \varpi)$ as the scalar $\chi(z \varpi)(\Delta)=z^{2}+2 z$. Note that $\rho=\varpi$ and $\chi(z \varpi) \equiv \chi\left(z^{\prime} \varpi\right)$ on $Z(\mathfrak{U} \mathfrak{g})=\mathbb{C}[\Delta]$, if and only if $z+z^{\prime}=-2$ - i.e., $z^{\prime} \varpi=s *(z \varpi)$. Similarly, $s * h=-h-2$, so:

$$
\beta^{\Pi}(\Delta)=(h+1)^{2}-1=((s * h)+1)^{2}-1=s * \beta^{\Pi}(\Delta) .
$$

\section{HARISH-ChandRa MOdUles}

We start our discussion of PRV2] with the main motivation: the works of Harish-Chandra. The representations studied by Parthasarathy, Ranga Rao, and Varadarajan are known today as (irreducible) admissible Harish-Chandra modules. They were first studied in the setting of real semisimple Lie groups by Harish-Chandra in [Har2, Har3].

2.1. For the better part of a century, and since the advent of quantum mechanics, mathematicians have been interested in unitary representations and harmonic analysis of locally compact (abelian) topological groups. One of the basic results in this direction is the Peter-Weyl Theorem, which says that every unitary (Hilbert space) representation of a compact group decomposes as a direct sum of finite-dimensional irreducible submodules. Given the correspondence between complex semisimple groups and compact groups (discovered by Weyl), the class of unitary representations of complex semisimple Lie groups $G$ and their maximal compact subgroups $K$ has been a subject of wide interest and research in the literature.

To explain the motivation for [PRV2], some notation is now needed. Given $G \supset K$ as above, $\mathfrak{k}=\operatorname{Lie}(K)$ is the compact form of $\mathfrak{g}=\operatorname{Lie}(G)$. Let $\mathfrak{g}^{\mathbb{C}}:=\operatorname{Lie}(G) \otimes_{\mathbb{R}} \mathbb{C}$ be the complexification of its Lie algebra; this is a complex semisimple Lie algebra that contains the reductive subalgebra $\mathfrak{k}^{\mathbb{C}}:=\operatorname{Lie}(K) \otimes_{\mathbb{R}} \mathbb{C}$. In his works cited above, Harish-Chandra initiated the study of a class of irreducible infinite-dimensional $G$-modules that was larger than the class of unitary $G$-modules 
(yet these modules were direct sums of finite-dimensional $K$-modules). Harish-Chandra constructed and studied these modules algebraically, via their correspondence to $\mathfrak{g}^{\mathbb{C}}$-modules (when $G$ has finite center). This correspondence was known for finite-dimensional modules, but he showed how to extend it to a deep and powerful theory of Banach-space representations of $G$.

More precisely, given a continuous Banach space $G$-representation $\pi$, whose restriction to $K$ contains every irreducible $K$-module with at most finite multiplicity (this is called "admissibility"), Harish-Chandra considered its subspace of $K$-finite vectors (i.e., the vectors that lie in finite-dimensional $K$-stable subspaces) - or more precisely, the $\mathfrak{k}^{\mathbb{C}}$-finite vectors. This subspace is called the infinitesimal representation associated to $\pi$. Two such Banach space representations are said to be infinitesimally equivalent if their infinitesimal representations are equivalent. (For instance, Harish-Chandra showed in Har2 that two irreducible unitary $G$-modules are equivalent if and only if they are infinitesimally equivalent.) One of the crown jewels of his work is the subquotient theorem [Har3], which says that every such admissible $V$ is infinitesimally equivalent to a subquotient of a Hilbert space representation of $G$ (the "principal series representations").

2.2. We now return to [PRV2], where the authors are interested in using Harish-Chandra's work to gain a deeper understanding of a special case of this situation: namely, when $G$ is already a complex group. (This setting was also studied earlier - by Weyl in relating complex and compact groups, but also by Gelfand and Naimark [GN], and Harish-Chandra as well.) By [Har3], it turns out that every $\mathfrak{k}^{\mathbb{C}}$-finite irreducible $G$-representation $V$ (with at most finite multiplicities) has an infinitesimal character. In other words, the center $Z\left(\mathfrak{U}\left(\mathfrak{g}^{\mathbb{C}}\right)\right)$ acts by scalars on it. As noted in PRV2, Va], the problem of describing the infinitesimal equivalence classes of irreducible Banach space $G$-representations (which are "admissible", hence equipped with an infinitesimal character) can now be reduced by Harish-Chandra's work, to describing the irreducible $\mathfrak{k}^{\mathbb{C}}$-finite representations - i.e., the so-called simple " $\left(\mathfrak{g}^{\mathbb{C}}, \mathfrak{k}^{\mathbb{C}}\right)$-modules" (or "( $\left.\mathfrak{g}^{\mathbb{C}}, K\right)$-modules"). Later in this section, we will mention certain prominent features from Harish-Chandra's approach in the real semisimple case, as we specialize them to the complex case in [PRV2].

Thus, the primary motivation in [PRV2] was to study the irreducible $G$-representations when $G$ is a complex semisimple group, by applying the methods and deep results from [Har2, Har3]. For instance, the authors are able to simplify Harish-Chandra's description of the closed subspaces of the principal series representations, which yield Banach (actually, Hilbert) space $G$ representations. Furthermore, the theory of minimal types developed in PRV2] helps obtain a deeper understanding of these simple $\left(\mathfrak{g}^{\mathbb{C}}, \mathfrak{k}^{\mathbb{C}}\right)$-modules.

We now introduce the setting of [PRV2]. If $G$ is a complex Lie group, then $\mathfrak{g}:=\operatorname{Lie}(G)$ is a complex Lie algebra, and $\operatorname{Lie}(K)$ is its compact (real) form. Thus as real Lie algebras, $\mathfrak{g}=\operatorname{Lie}(K) \oplus \sqrt{-1} \cdot \operatorname{Lie}(K)$ in the complex structure of $\mathfrak{g}$. The complexified pair $\left(\mathfrak{g}^{\mathbb{C}}, \mathfrak{k}^{\mathbb{C}}\right)$ is isomorphic to $(\mathfrak{g} \times \mathfrak{g}, \overline{\mathfrak{g}})$, where $\overline{\mathfrak{g}}$ is the diagonal copy of $\mathfrak{g}$ embedded in $\mathfrak{g} \times \mathfrak{g} 11$ Now the $\mathfrak{g} \times \mathfrak{g}$ modules of interest (studied by Harish-Chandra in general) are the ones that decompose into direct sums of finite-dimensional $\overline{\mathfrak{g}}$-modules with at most finite multiplicities.

Here is the precise framework studied in PRV2] (and henceforth in this article), stated here in a slightly more general setting.

Definition 2.1. Suppose $\mathfrak{g}$ is a complex reductive finite-dimensional Lie algebra contained in a complex Lie algebra $\widehat{\mathfrak{g}}$. Define the category $\mathcal{C}(\widehat{\mathfrak{g}}, \mathfrak{g})$ to be the full subcategory of $\widehat{\mathfrak{g}}$-modules, such that every object is isomorphic to a direct sum of finite-dimensional irreducible $\mathfrak{g}$-modules $\mathcal{D}$, each of which occurs with finite multiplicity. (This last condition is termed $\mathfrak{g}$-admissibility.)

\footnotetext{
${ }^{1}$ This is achieved using a conjugation $X \mapsto X^{c}$ of $\mathfrak{g}$ that fixes $\mathfrak{k}$. Thus, $\mathfrak{g}$ embeds inside $\mathfrak{g}^{\mathbb{C}}$ via: $X \mapsto\left(X^{c}, X\right)$, and when restricted to $\mathfrak{k}$, one obtains: $X \mapsto(X, X)$ - whence we get that $\mathfrak{k}^{\mathbb{C}}=\overline{\mathfrak{g}}$.
} 
Define $[V: \mathcal{D}]$ to be this multiplicity (which may be zero if no summand is isomorphic to $\mathcal{D}$ ); this integer does not depend on the direct sum decomposition of $V$. (Note that we assume $\mathcal{D} \neq 0$.)

If $V$ is in $\mathcal{C}(\widehat{\mathfrak{g}}, \mathfrak{g})$ and $\mathcal{D}$ is a (nonzero) simple $\mathfrak{g}$-module, the isotypical subspace $V_{\mathcal{D}}$ of $V$ is defined as the (finite-dimensional) span of all the $\mathfrak{g}$-submodules of $V$ that are isomorphic to $\mathcal{D}$. Clearly, the center $Z(\mathfrak{U} \widehat{\mathfrak{g}})$ preserves $V_{\mathcal{D}}$ for each $\mathcal{D}$, and hence acts locally finitely on $V$. Moreover, $[V: \mathcal{D}]$ then equals $\left[V_{\mathcal{D}}: \mathcal{D}\right]=\operatorname{dim} V_{\mathcal{D}} / \operatorname{dim} \mathcal{D}$.

2.3. Examples of Harish-Chandra modules in the literature. The goal of [PRV2] was to study the simple objects in the category $\mathcal{C}(\mathfrak{g} \times \mathfrak{g}, \overline{\mathfrak{g}})$. Before elaborating on their results, we remark that various families of Harish-Chandra modules have been widely studied in the literature. For example, Harish-Chandra modules are examples of integrable $\overline{\mathfrak{g}}$-modules - i.e., $\overline{\mathfrak{g}}$-modules where every vector is contained in a finite-dimensional $\overline{\mathfrak{g}}$-module. Here are some other examples; in them, we always assume that $\mathfrak{g}$ is semisimple (and complex).

Example 2.2. Suppose $\mathfrak{g}$ is semisimple and $\mathfrak{g}_{0}$ is its compact real form. Let $G_{0}$ be a compact Lie group with Haar measure $\mu$, such that $\mathfrak{g}_{0}=\operatorname{Lie}\left(G_{0}\right)$. Then by the Peter-Weyl Theorem, a dense subspace $V$ of $L^{2}\left(G_{0}, \mathbb{C}, \mu\right)$ is an object of $\mathcal{C}(\mathfrak{g}, \mathfrak{g})$. Moreover, $[V: V(\lambda)]=\operatorname{dim} V(\lambda)$ for all $\lambda \in \Lambda^{+}$.

Example 2.3. Recall that $\mathfrak{U} \mathfrak{g}$ is a direct sum of finite-dimensional $\mathfrak{g}$-modules, since every term in its standard filtration is. Is it also an object of $\mathcal{C}(\mathfrak{g}, \mathfrak{g})$ ? The answer is no - in fact, no finite-dimensional module occurs with finite nonzero multiplicity. To see this, note by Kostant's "separation of variables theorem" [Ko2] that $\mathfrak{U} \mathfrak{g}$ is free as a module (under multiplication) over its center:

$$
\mathfrak{U} \mathfrak{g} \cong \mathbb{H}(\mathfrak{g}) \otimes Z(\mathfrak{U} \mathfrak{g})
$$

where $\mathbb{H}(\mathfrak{g})$ is (isomorphic as a $\mathfrak{g}$-module to) the space of "harmonic polynomials on $\mathfrak{g}$ ", and is stable under the adjoint action of $\mathfrak{g}$ on $\mathfrak{U} \mathfrak{g}$. Thus, the multiplicity in $\mathfrak{U} \mathfrak{g}$ of every finite-dimensional module is either 0 or $\operatorname{dim} Z(\mathfrak{U} \mathfrak{g})$, which is infinite. In particular, $\mathfrak{U} \mathfrak{g}$ is not admissible.

However, $\mathbb{H}(\mathfrak{g})$ is indeed an object in $\mathcal{C}(\mathfrak{g}, \mathfrak{g})$; in fact, Kostant proved in $[\overline{K o 2}$ that $[\mathbb{H}(\mathfrak{g})$ : $V(\lambda)]=\operatorname{dim} V(\lambda)_{0}$ for all $\lambda \in \Lambda^{+}$. This is the starting point for another important contribution of [PRV2] to the theory of semisimple and affine (quantized) Lie algebras - the so-called "PRV determinants". We will discuss these in a later section.

Example 2.5. Simple finite-dimensional $\mathfrak{g} \times \mathfrak{g}$-modules are clearly in $\mathcal{C}(\mathfrak{g} \times \mathfrak{g}, \overline{\mathfrak{g}})$, by Weyl's Theorem of complete reducibility. This example is also the starting point for a result and a conjecture from [PRV2] (the "PRV Theorem" and "PRV conjecture"), that have since been extensively used and generalized in the literature. We address these in detail in the next section.

Example 2.6. The above example of $\rho_{n}$ for $\mathfrak{g}=\mathfrak{s l}_{2}(\mathbb{C})$ can be used to produce an object in $\mathrm{C}\left(\mathfrak{s l}_{2}(\mathbb{C}), \mathfrak{s l}_{2}(\mathbb{C})\right)$ as follows: the $\mathfrak{s l}_{2}(\mathbb{C})$-module

$$
\mathbb{C}[X, Y]=\bigoplus_{n \geq 0} P_{n}=\bigoplus_{n \geq 0} V(n)
$$

is clearly such an object.

Note that $P_{n}=V(n)=\operatorname{Sym}^{n-1}(V(1))$ for all $n \in \mathbb{N}$. Thus, $\mathbb{C}[X, Y]=\operatorname{Sym}(V(1))$. With this in mind, we can generalize the above example to $\mathfrak{g}=\mathfrak{s l}_{n}(\mathbb{C})$, as it acts on its simple module $\mathbb{C}^{n}$ (for $n>1$ ). Consider the modules $\operatorname{Sym}^{k}\left(\mathbb{C}^{n}\right) \subset\left(\mathbb{C}^{n}\right)^{\otimes k}$ for $k \geq 0$. Identifying a basis of $\mathbb{C}^{n}$ with commuting variables $X_{1}, \ldots, X_{n}$, it is not hard to show that as $\mathfrak{g}$-modules, $\operatorname{Sym}^{k}\left(\mathbb{C}^{n}\right)$ is precisely the space $P_{n, k}$ of homogeneous polynomials in $X_{1}, \ldots, X_{n}$ of total degree $k$, where $e_{i j}$ acts on $P_{n, k}$ as $X_{i} \partial_{j}$ for all $1 \leq i, j \leq n$ and all $k$. 
One can now check that $P_{n, k}$ is a simple module over $\mathfrak{s l}_{n}(\mathbb{C}) 2$. Since $n>1$, hence $\operatorname{dim} P_{n, k}=$ $\left(\begin{array}{c}k+n-1 \\ n-1\end{array}\right)$ is increasing in $k$. Thus the $P_{n, k}$ are non-isomorphic for fixed $n$, and so

$$
\mathbb{C}\left[X_{1}, \ldots, X_{n}\right]=\bigoplus_{k \geq 0} P_{n, k}=\operatorname{Sym}\left(\mathbb{C}^{n}\right)
$$

is indeed an object in $\mathcal{C}\left(\mathfrak{s l}_{n}(\mathbb{C}), \mathfrak{s l}_{n}(\mathbb{C})\right)$.

Example 2.7. If $\mathfrak{g}$ is semisimple and $\mathfrak{h}$ is the Cartan subalgebra of $\mathfrak{g}$, then $\mathfrak{C}(\mathfrak{g}, \mathfrak{h})$ is the category of (admissible) weight modules. There has been extensive research on the study and classification of irreducible (admissible) weight modules; see [Ma3 for more on this. We remark that Mathieu also studied Harish-Chandra modules in other settings in [Ma2]: the Virasoro algebra, the Cartan algebra, and the affine Kac-Moody algebras (as mentioned in the conclusion to loc. cit.).

Moreover, a very special family of admissible weight modules constitutes the objects of the Bernstein-Gelfand-Gelfand Category $\mathcal{O}$, which was introduced in [BGG]. A lot of research has been undertaken on the Category $\mathcal{O}$ in various settings in modern representation theory - including semisimple and Kac-Moody Lie algebras, the quantum groups associated with them, the Virasoro algebra, and more modern constructions such as rational Cherednik algebras, infinitesimal Hecke algebras, and $W$-algebras. In particular, for semisimple $\mathfrak{g}$, the classification of irreducible admissible weight modules (by work of Mathieu [Ma3] and others) as well as of primitive ideals (by work of Duflo [Du3] and others) reduces to the study of simple objects in $\mathcal{O}$. See [Hu2, Jo2, $\mathrm{Kh}, \mathrm{MP}]$ for additional references and results.

2.4. A key class of homomorphisms. We now outline Harish-Chandra's strategy for studying admissible irreducible $G$-representations, as it is used by Parthasarathy et al in the complex setting. Given $\mathfrak{g} \subset \widehat{\mathfrak{g}}$ as above, let $\Omega$ denote the centralizer of $\mathfrak{g}$ in $\mathfrak{U} \widehat{\mathfrak{g}}$. (This is denoted by $\mathfrak{O}$ in Va.) Then $Z(\mathfrak{U} \mathfrak{g})+Z(\mathfrak{U} \widehat{\mathfrak{g}}) \subset \Omega \subset \mathfrak{U} \widehat{\mathfrak{g}}$ is a chain of algebras. Now suppose $V$ is an object of $\mathcal{C}(\widehat{\mathfrak{g}}, \mathfrak{g})$, and $\mathcal{D}$ is a simple finite-dimensional $\mathfrak{g}$-module such that $[V: \mathcal{D}]=r>0$. Then $\mathcal{D} \cong V(\lambda)$ as finite-dimensional (and hence, highest-weight) $\mathfrak{g}$-modules, and $V_{\mathcal{D}} \cong V(\lambda) \otimes \mathbb{C}^{r}$ as a $\mathfrak{g}$-module (i.e., $\mathbb{C}^{r}$ is the multiplicity space). Multiplication by every $z \in \Omega$ preserves the highest weight space $\left(V_{\mathcal{D}}\right)_{\lambda}=V(\lambda)_{\lambda} \otimes \mathbb{C}^{r}$; this yields a representation $\eta_{V, \mathcal{D}}$ of $\Omega$ into $\mathbb{C}^{r}$. (This is called $\eta\left(\left(\nu^{0}\right), \pi\right)$ in [PRV2, where $\pi=V$ and $\left(\nu^{0}\right)=\mathcal{D}$.) Moreover $V_{\mathcal{D}}$ now decomposes as $\mathcal{D} \otimes \mathbb{C}^{r}$, under the joint action of $\mathfrak{g}$ and $\Omega$.

As a special case, suppose $r=1$. Then $\eta_{V, \mathcal{D}}$ is a homomorphism $: \Omega \rightarrow \mathbb{C}$. These homomorphisms are the key tools used in [PRV2] to study simple admissible Harish-Chandra modules, as we now explain.

Suppose $\widehat{\mathfrak{g}}=\mathfrak{g} \times \mathfrak{g} \supset \overline{\mathfrak{g}}$. In order to study simple objects in $\mathcal{C}(\mathfrak{g} \times \mathfrak{g}, \overline{\mathfrak{g}})$, the authors of [PRV2] follow the approach suggested by Harish-Chandra in [Har3]: if $r=[V: \mathcal{D}]>0$, then as above, $V_{\mathcal{D}} \cong \mathcal{D} \otimes \mathbb{C}^{r}$ under the joint action of $\mathfrak{U} \overline{\mathfrak{g}}$ and $\Omega$ - and moreover, the $\Omega$-representation $\eta_{V, \mathcal{D}}$ is simple. Now the following remarkable fact holds: the equivalence class of the representation $\eta_{V, \mathcal{D}}$ of $\Omega$ determines that of $V$, for every component $\mathcal{D}$ with $r>0$. More precisely, if $V, V^{\prime}$ are simple objects of $\mathcal{C}(\mathfrak{g} \times \mathfrak{g}, \overline{\mathfrak{g}})$ and $\mathcal{D}$ is a simple finite-dimensional $\overline{\mathfrak{g}}$-module such that $[V$ : $\mathcal{D}]+\left[V^{\prime}: \mathcal{D}\right]>0$, then

$$
[V: \mathcal{D}]=\left[V^{\prime}, \mathcal{D}\right]>0, \eta_{V, \mathcal{D}} \cong_{\Omega} \eta_{V^{\prime}, \mathcal{D}} \Longleftrightarrow V \cong V^{\prime}
$$

\footnotetext{
${ }^{2}$ See http://math.stackexchange.com/questions/120338 for the sketch of a proof.

${ }^{3}$ In Varadarajan's reminiscences [Va, he points out on Page (xii) that $\Omega$ is highly nonabelian, so that the first inclusion is not an equality in general.
} 
(See [LMC] for a generalization of this fact.) Thus, a "first approach" would be to fix various $\mathcal{D}$ and study the $\Omega$-modules $\eta_{V, \mathcal{D}}$ for all $V$ with $[V: \mathcal{D}]>0$. The authors remark in PRV2] that such an approach was not very fruitful and so a different method had to be adopted. Their contribution was to introduce and study the following notion.

Definition 2.9. Suppose $V$ is a simple object in $\mathcal{C}(\mathfrak{g} \times \mathfrak{g}, \overline{\mathfrak{g}})$ and $\lambda \in \Lambda^{+}$. We say that $\lambda$ (or $\left.V_{\overline{\mathfrak{g}}}(\lambda)\right)$ is a minimal type of $V$ if $\left[V: V_{\overline{\mathfrak{g}}}(\lambda)\right]>0$, and

$$
\left[V: V_{\overline{\mathfrak{g}}}(\mu)\right]>0 \Longrightarrow \lambda \in \mathrm{wt} V_{\overline{\mathfrak{g}}}(\mu) \text {. }
$$

It is clear that there is at most one minimal type for each $V$.

Now the strategy is as follows: first study a class of modules $V$ for which the minimal type $\mathcal{D}$ can be shown to exist. These are the irreducible finite-dimensional representations of $\mathfrak{g} \times \mathfrak{g}$, and it turns out that there is an explicit recipe to compute the homomorphism $\eta_{V, \mathcal{D}}$ in this case. This recipe involves $\eta_{V, \mathcal{D}}$ equalling a polynomial-valued homomorphism $\mathbf{h}^{\Pi^{\prime}}: \Omega \rightarrow P\left(\mathfrak{h}^{*} \times \mathfrak{h}^{*}\right)$, evaluated at some $\lambda \in \Lambda^{+}, \nu \in \Lambda$ - in other words, $\mathbf{h}^{\Pi^{\prime}}(-; \lambda, \nu): \Omega \rightarrow \mathbb{C}$. (This is explained in a later section.)

The authors then go on to explicitly construct a family $\left\{\widehat{\pi}_{\lambda, \nu}: \nu \in \Lambda, \lambda \in \mathfrak{h}^{*}\right\}$ of simple objects in $\mathcal{C}(\mathfrak{g} \times \mathfrak{g}, \overline{\mathfrak{g}})$, each of which has a minimal type $\bar{\nu}$ occurring with multiplicity $r=1$. (This family necessarily includes the finite-dimensional simple $\mathfrak{g} \times \mathfrak{g}$-modules, as we will see below.) The authors show that for each such $\lambda$ and $\nu$, the related "key homomorphism" $\eta_{\bar{\pi}_{\lambda, \nu}, \bar{\nu}}: \Omega \rightarrow \mathbb{C}$ turns out to be the same recipe $\mathbf{h}^{\Pi^{\prime}}$ as above, now evaluated at (more general points) $\lambda, \nu$. Thus, Equation (2.8) can be applied to discuss the classification of these modules $\widehat{\pi}_{\lambda, \nu}$. (Here and henceforth, we abuse notation and use $\eta_{\widehat{\pi}_{\lambda, \nu}, \bar{\nu}}$ to refer to $\left.\eta_{\widehat{\pi}_{\lambda, \nu}, V_{\overline{\mathfrak{g}}}(\bar{\nu}) \cdot}\right)$

Thus, the starting point for [PRV2] - and even earlier, for Varadarajan and Varadhan in 1963 for the special case of $\mathfrak{g}=\mathfrak{s l}_{n}(\mathbb{C})$ - was to prove the assertion that finite-dimensional irreducible $\mathfrak{g} \times \mathfrak{g}$-modules have minimal types. Note that such a simple module has highest weight in $\Lambda^{+} \times \Lambda^{+} \subset(\mathfrak{h} \times \mathfrak{h})^{*}$, so we can write it as $V(\lambda, \mu)$. It is clear that for all $X \in \mathfrak{g}$, its image in

$$
\overline{\mathfrak{g}} \subset \mathfrak{g} \times \mathfrak{g} \subset \mathfrak{U}(\mathfrak{g} \times \mathfrak{g})=\mathfrak{U} \mathfrak{g} \otimes \mathfrak{U} \mathfrak{g}
$$

is precisely $X \otimes 1+1 \otimes X$. Thus, restricting $V(\lambda, \mu)$ to $\overline{\mathfrak{g}}$ amounts to considering the module $V_{\overline{\mathfrak{g}}}(\lambda) \otimes V_{\overline{\mathfrak{g}}}(\mu)$. In other words, the study of the minimal type in this setting involves computing the direct summands of the tensor product - i.e., computing Clebsch-Gordan coefficients. This classical problem is the focus of the next section.

2.5. Digression on minimal type due to Vogan. We end this section with a few remarks on the notion of minimal type. The more widely accepted notion of minimal $K$-type (or lowest $K$ type) in the literature is due to Vogan [Vo], and differs from the above notion (in [PRV2]). More precisely, Vogan defines a weight $\lambda \in \Lambda^{+}$to be a lowest $K$-type for an admissible Harish-Chandra $(G, K)$-module $M$, if:

- $V(\lambda)$ is a $K$-submodule of $M$; and

- Among all $\mu$ such that $V(\mu)$ is a $K$-submodule of $M$, the quantity $(\mu+2 \rho, \mu+2 \rho)$ is minimized at $\mu=\lambda$.

One can now ask what is the relation between these two notions. Note that the definition due to Vogan guarantees existence of the minimal type (for irreducible admissible representations), but not uniqueness. In fact, uniqueness does not hold when $G$ is a (general) real group, such as $S L(2, \mathbb{R})$. However, uniqueness of the minimal $K$-type is guaranteed if $G$ is a complex group; see Zh2 for more details.

On the other hand, the definition in PRV2 guarantees uniqueness but not existence. If this version of the minimal type does exist, then it is necessarily a minimal type due to Vogan. This 
can be shown using the following generalization of [Hu1, Lemma 13.4.C] (whose proof is the same as that of loc. cit.), with $A=$ wt $V(\mu)$ for any finite-dimensional $K$-submodule $V(\mu)$ of $M$ :

Proposition 2.10. Suppose $A \subset \Lambda$ is $W$-stable, with highest weight $\mu$. (In other words, $A \subset$ $\mu-\mathbb{Z}_{\geq 0} \Pi$.) Fix $\lambda \in A$ and $0<c \in \mathbb{R}$. Then $\mu \in \Lambda^{+}$and $A$ is finite; moreover,

$$
(\lambda+c \rho, \lambda+c \rho) \leq(\mu+c \rho, \mu+c \rho),
$$

with equality if and only if $\lambda=\mu$.

\section{Tensor products, minimal types, and the (K)PRV conjecture}

In this section, we discuss [PRV2, Section 2.2], which contains several results, as well as a related conjecture, that have been extremely influential on subsequent research in the field. These results and the conjecture have to do with the classical question of computing tensor product multiplicities. Although the primary motivation of Parthasarathy et al was to study tensor products in order to prove the existence and uniqueness of minimal types, some of these statements have been subsequently generalized and have contributed to several aspects of the multiplicity problem. We will list some of the relevant papers and results presently.

3.1. Tensor product multiplicities and the PRV Theorem. We start by recalling the notion of Littlewood-Richardson coefficients. By Weyl's theorem of complete reducibility, given $\lambda, \mu \in \Lambda^{+}$, we can decompose

$$
V(\lambda) \otimes V(\mu)=\bigoplus_{\nu \in \Lambda^{+}} m_{\lambda, \mu}^{\nu} V(\nu)
$$

where the multiplicities $m_{\lambda, \mu}^{\nu}=m_{\mu, \lambda}^{\nu}$ are the coefficients in question, also known as tensor product multiplicities. (In the rest of this article, we will abuse notation and denote $V_{\overline{\mathfrak{g}}}(\lambda)$ by $V(\lambda)$.) If $m_{\lambda, \mu}^{\nu}>0$, we say that $V(\nu)$ is a component of $V(\lambda) \otimes V(\mu)$. For example, $V(\lambda+\mu)$ is always a component, generated by $V(\lambda)_{\lambda} \otimes V(\mu)_{\mu}$, and $m_{\lambda, \mu}^{\lambda+\mu}=1$ for all $\lambda, \mu \in \Lambda^{+}$.

The determination of the multiplicities $m_{\lambda, \mu}^{\nu}$ is a longstanding open problem in the literature - as is the simpler problem of computing whether or not $m_{\lambda, \mu}^{\nu}$ is positive. Efforts to answer these questions have been ongoing since even before [PRV2]. For instance, in his famous paper [Ko1], Kostant proved his multiplicity formula, and also showed a necessary condition for $V(\nu)$ to be a component: it must be of the form $\nu=\lambda+\mu_{1} \in \Lambda^{+}$for some $\mu_{1} \in \operatorname{wt}(V(\mu))$. Moreover, $m_{\lambda, \mu}^{\nu} \leq \operatorname{dim} V(\mu)_{\nu-\lambda}$. By work [St] of Steinberg (using Kostant's multiplicity formula), the following was also known:

$$
m_{\lambda, \mu}^{\nu}=\sum_{w \in W} \operatorname{sn}(w) \operatorname{dim} V(\mu)_{w * \nu-\lambda}=\sum_{w, w^{\prime} \in W} \operatorname{sn}(w) \operatorname{sn}\left(w^{\prime}\right) \mathcal{P}\left(w^{\prime}(\mu+\rho)-w(\nu+\rho)+\lambda\right) .
$$

Here, $\mathcal{P}: \Lambda^{+} \rightarrow \mathbb{N}$ is the Kostant partition function (which is also defined to be zero on $\Lambda \backslash \Lambda^{+}$), and sn :W $\rightarrow\{ \pm 1\}$ is the sign homomorphism, which is -1 on all simple reflections $s_{i}$. Steinberg's results imply $\mathrm{Ku} 5$ ] that if $\left(\lambda+\mu^{\prime}\right)\left(h_{i}\right) \geq-1$ for all $\mu^{\prime} \in \operatorname{wt}(V(\mu))$ and $i \in I$, then $m_{\lambda, \mu}^{\nu}=\operatorname{dim} V(\mu)_{\nu-\lambda}$. Kostant had shown a special case of this result in [Ko1, where he assumed that $\left(\lambda+\mu^{\prime}\right)\left(h_{i}\right) \geq 0$.

In PRV2], the following multiplicity formula is proved. Given $\mu, \nu \in \Lambda^{+}$and $\gamma \in \mathfrak{h}^{*}$, define:

$$
\begin{aligned}
V^{+}(\mu ; \gamma, \nu) & :=\left\{v \in V(\mu)_{\gamma}: e_{i}^{\nu\left(h_{i}\right)+1} v=0 \forall i \in I\right\}, \\
V^{-}(\mu ; \gamma, \nu) & :=\left\{v \in V(\mu)_{\gamma}: f_{i}^{\nu\left(h_{i}\right)+1} v=0 \forall i \in I\right\} .
\end{aligned}
$$


Theorem 3.1 ([PRV2] $)$. For all $\lambda, \mu, \nu \in \Lambda^{+}$,

$$
m_{\lambda, \mu}^{\nu}=\operatorname{dim} V^{+}(\mu ; \nu-\lambda, \lambda)=\operatorname{dim} V^{+}\left(\nu ; \lambda+w_{\circ} \mu,-w_{\circ} \mu\right) .
$$

Now given $\gamma \in \mathfrak{h}^{*}, \operatorname{dim} V^{+}(\mu ; \gamma, \nu)=\operatorname{dim} V^{-}\left(\mu ; w_{\circ} \gamma,-w_{\circ} \nu\right)$.

Here is a typical application of this result, which shows how to compute multiplicities.

Example 3.2. Suppose $\lambda, \nu \in \Lambda^{+}$. If $\left[V(\lambda) \otimes V(\lambda)^{*}: V(\nu)\right]>0$, then $\lambda-w_{\circ} \lambda-\nu \in \mathbb{Z}_{\geq 0} \Pi$ by Kostant's results, which implies that $\nu \in \Lambda^{+} \cap \mathbb{Z} \Pi$. For every such $\nu$, Theorem 3.1 now says:

$$
m_{\lambda,-w_{\circ} \lambda}^{\nu}=\operatorname{dim} V^{+}(\nu ; 0, \lambda):=\operatorname{dim}\left\{v \in V(\nu)_{0}: e_{i}^{\lambda\left(h_{i}\right)+1} v=0 \forall i \in I\right\} .
$$

Thus if $\lambda\left(h_{i}\right)$ is large enough for all $i$ (e.g., $\lambda=n \rho$ for $n \gg 0$ ), then

$$
\left[V(\lambda) \otimes V(\lambda)^{*}: V(\nu)\right]=\operatorname{dim} V(\nu)_{0}>0, \quad \forall \nu \in \Lambda^{+} \cap \mathbb{Z} \Pi .
$$

where the last inequality follows by a result from [Hal], used below to prove Proposition 3.6.

The advantage of the "PRV Theorem" 3.1 over some of the earlier formulae in the literature is that it calculates the multiplicities directly and without cancellation. For instance, note that the above result of Steinberg involves a double summation over the Weyl group - and cancellations of terms - and hence is not suitable for practical computations. Several years prior to PRV2, Brauer had proposed another such formula in $[\mathrm{Br}$; it is similar to a result of Klimyk in [Kl], which appeared in the same year as [PRV2]. The result is stated as Exercise 24.9 in [Hu1]. It computes the multiplicities $m_{\lambda, \mu}^{\nu}$ as sums of dimensions $\operatorname{dim} V(\mu)_{\mu^{\prime}}$, but with coefficients of \pm 1 and 0 , which again implies the need to perform cancellation (of formal characters).

It is mentioned in PRV2 that Kostant had obtained Theorem 3.1 previously but had not published it; for a historical account of this result, see [Ko4].

The PRV Theorem 3.1 has been widely used and generalized in the literature. In [CP], Chari and Pressley extend a special case of this result to show that simple integrable modules over affine Lie algebras are quotients of tensor products. In [Jo2], Joseph studies a similar result for a general symmetrizable (quantum) Kac-Moody Lie algebra - as does Mathieu in [Ma1]. Among other applications, Young and Zegers start from Theorem 3.1 in [YZ] and relate Dorey's rule to $q$-characters of fundamental representations of quantum affine algebras of type ADE. Panyushev and Yakimova study variants and consequences of the result in [PY].

From a personal viewpoint, the author has used the PRV Theorem in his paper [CKR] with Chari and Ridenour, to provide examples of families of finite and infinite-dimensional Koszul algebras which naturally arise out of module categories over semidirect products $\mathfrak{g} \ltimes V(\lambda)$. The result was also used by Chari and Greenstein [CG1, CG2] in the study of representations of the truncated current algebra $\mathfrak{g}[t] /\left(t^{2}\right)$, as well as in other works of Chari and her collaborators, and of Greenstein. These papers have applications in the study of Kirillov-Reshetikhin modules over quantum affine algebras.

3.2. Minimal type. We again start by considering the decomposition of the tensor product into its simple module components. Consider the example where $\mathfrak{g}=\mathfrak{s l}_{2}(\mathbb{C})$ and $0 \leq \mu \leq \lambda \in \mathbb{Z}_{\geq 0}$. (Once again, we abuse notation and use $\lambda \in \mathbb{Z}$ to refer to $\lambda \cdot \varpi \in \Lambda$.) Recall the well-known Clebsch-Gordan formula for $\mathfrak{s l}_{2}(\mathbb{C})$ :

$$
V(\lambda) \otimes V(\mu)=V(\lambda+\mu) \oplus V(\lambda+\mu-2) \oplus \cdots \oplus V(\lambda-\mu)
$$

We see that there are two distinguished components in this direct sum:

\footnotetext{
${ }^{4}$ See also: http://mathoverflow.net/questions/85593/
} 
- The "largest" component is $V(\lambda+\mu)$, in that every highest weight occurring on the right, belongs to wt $V(\lambda+\mu)$. This is called the Cartan component or the maximal type, and occurs with multiplicity 1 . It is generated by the one-dimensional vector space $V(\lambda)_{\lambda} \otimes V(\mu)_{\mu}$, which is the tensor product of the two highest weight spaces.

- The "smallest" component is $V(\lambda-\mu)$, in that $\lambda-\mu$ is a weight of every summand occurring on the right. This is called the PRV component (after the authors of [PRV2] or the minimal type, and it also occurs with multiplicity 1.

It is reasonable to ask if these results extend to all semisimple $\mathfrak{g}$. Remarkably, the authors of PRV2 found the answer of this question to be positive! To understand it, one must first make sense of what the minimal type is for general $\mathfrak{g}$. Note above that we could have interchanged $\lambda$ and $\mu$, since the tensor product is "commutative" (i.e., the Hopf algebra $\mathfrak{U} \mathfrak{g}$ is cocommutative). Thus, to choose the minimal type, one chooses the dominant integral weight from among $\{\lambda-$ $\mu, \mu-\lambda\}=W\left(\lambda+w_{\circ} \mu\right)$. Supporting evidence is obtained from Theorem 3.1, where if $\lambda+w_{\circ} \mu \in$ $\Lambda^{+}$, then substituting it for $\nu$ yields:

$$
m_{\lambda, \mu}^{\lambda+w_{\circ} \mu}=\operatorname{dim} V^{+}\left(\lambda+w_{\circ} \mu ; \lambda+w_{\circ} \mu,-w_{\circ} \mu\right)=1 .
$$

This led Varadarajan and Varadhan to generalize the existence of the minimal type to $\mathfrak{g}=\mathfrak{s l} \mathfrak{l}_{n}(\mathbb{C})$ for all $n$, while they were at the Indian Statistical Institute, Kolkata. (See [Va] for a very nice historical account of the development of [PRV2].) Subsequently in [PRV2], the authors extended the result to arbitrary semisimple $\mathfrak{g}$, and obtained the previously sought-for existence and unique multiplicity of the minimal type. Here is their result.

Theorem 3.5 ([PRV2]). Suppose $\mathfrak{g}$ is semisimple, and $\lambda, \mu \in \Lambda^{+}$. Given $\nu \in \Lambda^{+}$, define $\bar{\nu}$ to be the unique $W$-translate of $\nu$ that lies in $\Lambda^{+}$. Then $m_{\lambda, \mu}^{\overline{\lambda+w_{\circ} \mu}}=1$. Moreover,

$$
m_{\lambda, \mu}^{\nu}>0 \Longrightarrow \overline{\lambda+w_{\circ} \mu} \in \mathrm{wt} V(\nu) .
$$

(More generally - say by the result from [KLV] stated in the next proof below - wt $V\left(\overline{\lambda+w_{\circ} \mu}\right) \subset$ wt $V(\nu)$ for all such $\nu$.) Thus, the sought-for minimal type exists and possesses the desired properties. We will see later, how this leads to the construction of interesting polynomial maps and infinite-dimensional Banach space representations of $G$.

For completeness, we remark that the "maximal type" also exists in general:

Proposition 3.6. If $\mathfrak{g}$ is semisimple and $\lambda, \mu \in \Lambda^{+}$, then $m_{\lambda, \mu}^{\lambda+\mu}=1$. Moreover,

$$
m_{\lambda, \mu}^{\nu}>0 \Longrightarrow \nu \in \operatorname{wt} V(\lambda+\mu) \text {. }
$$

More generally, wt $V(\lambda) \otimes V(\mu)=$ wt $V(\lambda+\mu)$.

Inductively, wt $\otimes_{i=1}^{n} V\left(\lambda_{i}\right)=$ wt $V\left(\sum_{i} \lambda_{i}\right)$ if all $\lambda_{i} \in \Lambda^{+}$.

Proof. We only show that wt $V(\lambda) \otimes V(\mu) \subset$ wt $V(\lambda+\mu)$. Note that wt $V(\lambda) \otimes V(\mu)=$ $\bigcup_{\nu}$ wt $V(\nu)$, where we run over all $\nu \in \Lambda^{+}$such that $m_{\lambda, \mu}^{\nu}>0$. Now from Kostant's results mentioned above, every such $\nu$ is of the form $\lambda+\mu^{\prime}$, where $\mu^{\prime} \in$ wt $V(\mu)$. Hence it suffices to prove that

$$
\mu^{\prime} \in \text { wt } V(\mu), \lambda+\mu^{\prime} \in \Lambda^{+} \Longrightarrow \text { wt } V\left(\lambda+\mu^{\prime}\right) \subset \text { wt } V(\lambda+\mu) .
$$

We now quote a result from [KLV], which says that given $\lambda, \mu \in \Lambda^{+}, \lambda-\mu \in \mathbb{Z}_{\geq 0} \Pi$ if and only if $\operatorname{conv}(W \mu) \subset \operatorname{conv}(W \lambda)$, where conv denotes the convex hull. Applying this with $\mu \rightsquigarrow \lambda+\mu^{\prime}, \lambda \rightsquigarrow \lambda+\mu, \operatorname{conv}\left(W\left(\lambda+\mu^{\prime}\right)\right) \subset \operatorname{conv}(W(\lambda+\mu))$. Now given $\nu^{\prime} \in \operatorname{wt} V\left(\lambda+\mu^{\prime}\right)$, it is clear that $(\lambda+\mu)-\nu^{\prime} \in \mathbb{Z}_{\geq 0} \Pi$. Recall [Hal, Theorem 7.41], which says that for all $\lambda \in \Lambda^{+}$, wt $V(\lambda)=(\lambda-\mathbb{Z} \Pi) \cap \operatorname{conv}(W \lambda)$. Applying this first with $\lambda \rightsquigarrow \lambda+\mu^{\prime}$ and then with $\lambda \rightsquigarrow \lambda+\mu$, we get that $\nu^{\prime} \in \operatorname{conv}\left(W\left(\lambda+\mu^{\prime}\right)\right) \subset \operatorname{conv}(W(\lambda+\mu))$, so $\nu^{\prime} \in$ wt $V(\lambda+\mu)$ as desired. 
3.3. The (K)PRV conjecture and generalized PRV components. We now discuss a vast generalization of Theorem 3.5, which was conjectured by Parthasarathy et al, extended by Kostant and refined by Verma, and proved by Kumar in [Ku1, Ku3]. The "PRV Conjecture" has been the subject of much study and numerous papers in the literature, and continues to attract interest, as we point out below.

To state the conjecture, recall the following facts from above: given $\lambda, \mu \in \Lambda^{+}$,

- $m_{\lambda, \mu}^{\lambda+1 \cdot \mu}=1$.

- Equation (3.4) says: $\lambda+w_{\circ} \mu \in \Lambda^{+} \Longrightarrow m_{\lambda, \mu}^{\lambda+w_{\circ} \mu}=1$.

There is a common generalization of these assertions to arbitrary $w \in W$, which is mentioned in [Ku5, PY]. Namely, given $\lambda, \mu \in \Lambda^{+}$and $w \in W$,

$$
\lambda+w \mu \in \Lambda^{+} \Longrightarrow m_{\lambda, \mu}^{\lambda+w \mu}=1 .
$$

It is natural to ask what happens when $\lambda+w \mu \notin \Lambda^{+}$. In light of Theorem 3.5, a natural guess would be to ask if $m_{\lambda, \mu}^{\overline{\lambda+w \mu}}=1$, or at least, if this multiplicity is positive. This is known as the $P R V$ Conjecture in the literature.

Kostant significantly strengthened the PRV conjecture in the following way. Recall that the formal character of each finite-dimensional module $V(\lambda)$ is $W$-invariant, which implies that for all $w \in W, \operatorname{dim} V(\lambda)_{w \lambda}=1$. Suppose $v_{w \lambda}$ and $v_{w \mu}^{\prime}$ are nonzero vectors that span the "extremal weight spaces" $V(\lambda)_{w \lambda}$ and $V(\mu)_{w \mu}$ respectively, for all $\lambda, \mu \in \Lambda^{+}$and $w \in W$. It is then clear that $v_{\lambda} \otimes v_{\mu}^{\prime}$ generates the copy of the "maximal type" $V(\lambda+\mu)$ inside $V(\lambda) \otimes V(\mu)$. Now consider the minimal type: is it generated by $v_{\lambda} \otimes v_{w_{\circ} \mu}^{\prime}$ ? The answer is no: in fact, this vector generates the entire module! In other words, $\mathfrak{U} \mathfrak{g}\left(v_{\lambda} \otimes v_{w_{\circ} \mu}^{\prime}\right)=V(\lambda) \otimes V(\mu)$. Moreover, Theorem 3.5 says that exactly one copy of $V\left(\overline{\lambda+w_{\circ} \mu}\right)$ sits in it.

It is now possible to generalize both of these statements. Given any $w \in W$, consider the $\mathfrak{g}$-submodule generated by $v_{\lambda} \otimes v_{w \mu}^{\prime}$. Does it contain a (unique) copy of $V(\overline{\lambda+w \mu})$ ? This is the subject of the KPRV conjecture, which was formulated by Kostant and proved by Kumar in Ku1] in the semisimple case.

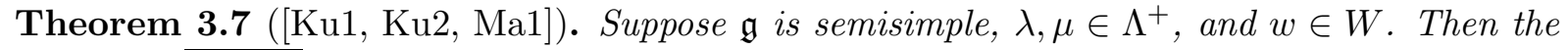
module $V(\overline{\lambda+w \mu})$ appears with multiplicity 1 in the submodule $\mathfrak{U} \mathfrak{g}\left(v_{\lambda} \otimes v_{w \mu}^{\prime}\right)$ of $V(\lambda) \otimes V(\mu)$.

Note that a part of Theorem 3.5 is just the special case $w=w_{\circ}$ of this result. Moreover, the components $V(\overline{\lambda+w \mu})$ are known as generalized PRV components.

The KPRV conjecture was also extended to symmetrizable Kac-Moody Lie algebras by Mathieu. Given a symmetrizable generalized Cartan matrix $A$, one can again define the associated Kac-Moody Lie algebra $\mathfrak{g}(A)$ over a field $k$. When char $k=0$, one defines the above notions of dominant integral weights $\Lambda^{+}$and $\bar{\lambda}$, as well as simple highest weight $\mathfrak{g}(A)$-modules $L(\lambda)$ corresponding to any weight $\lambda$. In [Ma1], Mathieu defined an associated Kac-Moody group over an arbitrary field $k$ (after earlier work by Kac, Moody, Peterson, and Tits) using the formalism of ind-schemes. His work led him to prove Theorem 3.7 for $\mathfrak{g}(A)$. (Kumar also proved this case under the assumption that $\lambda$ is regular, in $\mathrm{Ku} 2$.)

Other proofs of the (K)PRV conjecture have since appeared in the literature (this is from [Ku5]). For example, Polo had proved the PRV conjecture in type $A$ in [Po]. Rajeswari [Ra] gave a proof for classical $\mathfrak{g}$ using Standard Monomial Theory; Littelmann did so using his LS-path model (which generalizes the Littlewood-Richardson rule using tableaux for $\mathfrak{g l}(n)$, to symmetrizable Kac-Moody algebras - see [Li]); and Lusztig's work on the intersection homology of generalized Schubert varieties associated to affine Kac-Moody groups also provides a proof. 
Here is another related result. Note that if $w^{\prime} \leq w$ in the Bruhat order, then $\mathfrak{U} \mathfrak{g}\left(v_{\lambda} \otimes v_{w^{\prime} \mu}^{\prime}\right) \subset$ $\mathfrak{U} \mathfrak{g}\left(v_{\lambda} \otimes v_{w \mu}^{\prime}\right)$. This follows inductively from the case when $w=s_{i} w^{\prime}>w^{\prime}$, which is proved inside the module $V_{i}\left(\left(w^{\prime} \mu\right)\left(h_{i}\right)\right)$ over the " $\alpha_{i}$-copy" of $\mathfrak{s l}_{2}(\mathbb{C})$, by showing that $v_{\lambda} \otimes v_{w^{\prime} \mu}^{\prime}=$ $e_{i}^{\left(w^{\prime} \mu\right)\left(h_{i}\right)}\left(v_{\lambda} \otimes v_{w \mu}^{\prime}\right)$. One can now ask if the component $V(\overline{\lambda+w \mu})$ occurs in $\mathfrak{U} \mathfrak{g}\left(v_{\lambda} \otimes v_{w^{\prime} \mu}^{\prime}\right)$ for some $w^{\prime}<w$, or if $\mathfrak{U} \mathfrak{g}\left(v_{\lambda} \otimes v_{w \mu}^{\prime}\right)$ is the "first time" that it occurs in $V(\lambda) \otimes V(\mu)$.

Proposition 3.8 ([Ku1]). Given regular $\lambda, \mu \in \Lambda^{+}$(i.e., $\left.\lambda\left(h_{i}\right), \mu\left(h_{i}\right)>0 \forall i \in I\right)$ and $w^{\prime}<w$ in the Bruhat order on $W$, the $\mathfrak{g}$-module $V(\overline{\lambda+w \mu})$ does not occur in $\mathfrak{U} \mathfrak{g}\left(v_{\lambda} \otimes v_{w^{\prime} \mu}^{\prime}\right)$.

We end this part by mentioning two further directions in which the (original) PRV conjecture has been generalized very recently. Suppose $G \subset \widehat{G}$ are complex connected reductive groups, such that $\widehat{W}$ is the Weyl group of $\widehat{G}$ and various subgroups are "compatible" with the inclusion $: G \hookrightarrow \widehat{G}$ (e.g., $B \subset \widehat{B}, T \subset \widehat{T}, W \subset \widehat{W}$ ). Given a dominant integral weight $\widehat{\lambda}$ for $\widehat{G}$ and $\widehat{w} \in \widehat{W}$, does the simple highest-weight (finite-dimensional) module $V_{G}(\overline{\rho(\widehat{w} \widehat{\lambda})})$ with extremal weight $\rho(\widehat{w} \widehat{\lambda})$ occur inside $V_{\widehat{G}}(\widehat{\lambda})$ (upon restricting this to $G$ )? Here, $\rho$ is the restriction of a weight from $\widehat{G}$ to $G$. For example, the classical PRV conjecture uses $\widehat{G}=G \times G$ containing the diagonal copy of $G$, and

$$
\widehat{W}=W \times W, \quad \widehat{\lambda}=(\lambda, \mu), \quad \widehat{w}=(1, w), \quad \rho(\lambda, \mu)=\lambda+\mu .
$$

The above question is addressed in great detail for more general pairs $G \subset \widehat{G}$ in the recent papers [MPR1, MPR2], under the assumption that $\widehat{G} / G$ is "spherical of minimal rank".

Finally, Hayashi has proved a quantum counterpart of the PRV conjecture in [Hay] in the context of fusion rules for $\widehat{\mathfrak{s l}_{3}}(\mathbb{C})$ and the moduli space of $S U(3)$-flat connections on a pair of pants. These references are intended to reinforce upon the reader that the PRV conjecture is an extremely well-studied result, with connections to several other settings in representation theory and beyond.

3.4. Tensor product multiplicities, revisited. We now return to the original question in this section, of computing Littlewood-Richardson coefficients. As shown above, several results and formulae have been proposed over the years. Additionally, various other approaches have appeared more recently in the literature. To name but a few: Littelmann's LS-path model, Lusztig's approach using canonical bases, and Kashiwara's use of crystals. See [BZ, Ka, Ku5, $\mathrm{Li}, \mathrm{Lu}]$ for references and results.

Recall that the basic questions involving tensor product multiplicities are: (a) when are the $m_{\lambda, \mu}^{\nu}$ positive, and (b) computing the $m_{\lambda, \mu}^{\nu}$. Results by Kostant, or the PRV conjecture, address the first question, while the PRV theorem and results by Brauer and Steinberg discuss the second one. As the above references and related results show, the work PRV2] has had quite an influential contribution in this regard.

We conclude this section with a few additional results in this direction (see [Ku5]) that exhibit new components, or in some cases, even obtain a complete decomposition of the tensor product. The first is a refinement of the above PRV conjecture. One can ask if $m_{\lambda, \mu}^{\overline{\lambda+w \mu}}=1$ for all $w$, since it is so for $w=1, w_{\circ}$ from above. This claim turns out to be false - in fact, Verma produced counterexamples for every $\mathfrak{g}$ of rank 2 (i.e., $|\Pi|=2$ ), by choosing $\lambda=\mu=\rho=\sum_{i \in I} \varpi_{i}$.

This led Verma to refine the PRV conjecture as follows. The refined statement was also proved by Kumar. 
Theorem 3.9 ([Ku3]). Given $\lambda, \mu \in \Lambda^{+}$and $w \in W$, define $W_{\lambda}$ to be the stabilizer subgroup of $\lambda$ in $W$, and the map $\eta: W_{\lambda} \backslash W / W_{\mu} \rightarrow \Lambda^{+}$via: $\eta\left(W_{\lambda} w W_{\mu}\right)=\overline{\lambda+w \mu}$. Then $m_{\lambda, \mu}^{\overline{\lambda+w \mu}} \geq$ $\# \eta^{-1}\left(\eta\left(W_{\lambda} w W_{\mu}\right)\right)$.

Of course, if $\lambda, \mu$ are both regular (i.e., $(\lambda, \alpha)$ and $(\mu, \alpha)$ are both nonzero for all roots $\alpha$ ), then $W_{\lambda}=W_{\mu}=\{1\}$.

Second, a related result from [Ku3] is able to determine all the multiplicities when wt $V(\mu)=$ $W \mu$ is a single orbit (i.e., $\mu$ is minuscule). In this case,

$$
V(\lambda) \otimes V(\mu) \cong \bigoplus_{\bar{w} \in W / W_{\mu}: \lambda+w \mu \in \Lambda^{+}} V(\lambda+w \mu)
$$

where each factor occurs with multiplicity 1 . There are exactly $\# W_{\lambda} \backslash W / W_{\mu}$ components.

Kumar also shows the following result in [Ku4]: suppose $\beta \in R^{+}$is such that $\lambda+\mu-\beta \in \Lambda^{+}$, and such that $\beta-\alpha_{i} \notin R^{+} \cup\{0\}$ whenever $\lambda\left(h_{i}\right)$ or $\mu\left(h_{i}\right)=0$. Then $m_{\lambda, \mu}^{\lambda+\mu-\beta}>0$. A similar result can be found in the recent work [MPR1], where the authors demonstrate new components of the form $w_{1} \lambda+w_{2} \mu-k \alpha_{i}$ for some $w_{1}, w_{2} \in W$ and $i \in I$.

Finally, Dimitrov and Roth have worked with restrictions of line bundles from the square $G / B \times G / B$ of the flag variety to the diagonally embedded copy. (Here, $G$ is a connected reductive algebraic group with $\operatorname{Lie}(G)=\mathfrak{g}$, and, $B \subset G$ is a Borel $\operatorname{subgroup}$ with $\operatorname{Lie}(B)=\mathfrak{h} \oplus \mathfrak{n}^{+}$; Kumar's proofs in [Ku1] involved a study of similar objects.) They study special components $V(\nu)$ of the tensor product modules $V(\lambda) \otimes V(\mu)$, that arise out of cohomological reasons. The authors mention in $[\mathrm{DR}]$ that these cohomological components automatically turn out to be generalized PRV components satisfying: $m_{k \lambda, k \mu}^{k \nu}=1$ for all $k \in \mathbb{N}$. They go on to prove the converse implication when $G$ is a classical group, as well as in other cases.

\section{Irreducible Banach SPACE RePRESEntations}

We now continue the discussion prior to the preceding section, about constructing irreducible $\overline{\mathfrak{g}}$-admissible Banach (actually, Hilbert) space representations of $G$. In [PRV2], having proved that finite-dimensional $\mathfrak{g} \times \mathfrak{g}$-modules have minimal types, the authors proceed to construct other such representations (possibly infinite-dimensional), in a completely different manner. Their construction depends heavily on the work of Harish-Chandra [Har1]-[Har4]. These representations $\widehat{\pi}_{\lambda, \nu}$ are defined on subquotients of a Hilbert space.

4.1. The work of Harish-Chandra. In order to outline the construction of these $\mathfrak{g}$-modules by Harish-Chandra and by Parthasarathy et al, additional notation is needed. Let $K \subset G$ be the maximal compact subgroup of a complex connected semisimple Lie group, and let $\mathfrak{h}_{0} \subset \operatorname{Lie}(K)$ be a Cartan subalgebra. Now define $M:=\exp \left(\sqrt{-1} \cdot \mathfrak{h}_{0}\right) \subset K$ to be the corresponding Cartan subgroup. For each $\nu \in \Lambda$, define $\sigma_{\nu}$ to be the unique character of $M$ that $\operatorname{sends} \exp (\sqrt{-1} \cdot h)$ to $\exp (\sqrt{-1} \cdot \nu(h))$ for all $h \in \mathfrak{h}_{0}$. Then $\nu \mapsto \sigma_{\nu}$ is an isomorphism of $(\Lambda,+)$ onto the character group $\widehat{M}$ of $M$.

Now let $\mathfrak{H}:=L^{2}(K, \mathbb{C}, \mu)$, where $\mu$ denotes the (normalized) Haar measure on the compact group $K$. This is a representation of $M$ under the right-regular action: $(m \cdot f)(k):=f\left(\mathrm{~km}^{-1}\right)$. Given $\nu \in \Lambda$, define the $\nu$-weight subspace of $\mathfrak{H}$ as follows:

$$
\mathfrak{H}(\nu):=\left\{f \in \mathfrak{H}: m \cdot f=\sigma_{-\nu}(m) f \forall m \in M\right\} .
$$

Then $\mathfrak{H}$ decomposes as the direct sum of the $\mathfrak{H}(\nu)$ over all $\nu \in \Lambda$. Moreover, given $\xi \in \mathfrak{h}^{*}$, Harish-Chandra had previously defined and studied a $G$-module structure $\pi_{\xi}$ on $\mathfrak{H}$ in [Har2]Har4]. It turns out that every $\mathfrak{H}(\nu)$ is a submodule of $\mathfrak{H}$ under this structure; define $\pi_{\xi, \nu}$ to be this representation. Here are some of the properties of these modules that are used in [PRV2]. 
Theorem 4.1 (Harish-Chandra). Fix $\xi \in \mathfrak{h}^{*}$ and $\nu \in \Lambda$.

(1) For all $\mu \in \Lambda^{+},\left[\pi_{\xi, \nu}: V_{\overline{\mathfrak{g}}}(\mu)\right]=\operatorname{dim} V_{\overline{\mathfrak{g}}}(\mu)_{\nu}$. In particular, $\left[\pi_{\xi, \nu}: V_{\overline{\mathfrak{g}}}(\bar{\nu})\right]=1$.

(2) The representation $\pi_{\xi, \nu}$ has an infinitesimal character (of $Z(\mathfrak{U}(\mathfrak{g} \times \mathfrak{g}))$ ).

(3) $\pi_{\xi, \nu}$ possesses a distributional character $\Theta_{\xi, \nu}$, which is a locally summable function that is analytic on the dense open subset of regular points of $G$. Moreover, $\Theta_{\xi, \nu}=\Theta_{\xi^{\prime}, \nu^{\prime}}$ if and only if there exists $w \in W$ such that $\xi^{\prime}=w \xi, \nu^{\prime}=w \nu$.

(4) If $\xi$ is restricted to lie in the real subspace $\mathfrak{R}$ of all weights that take purely imaginary values on $\mathfrak{h}_{0}$, then $\pi_{\xi, \nu}$ is always unitary, and almost always irreducible, say whenever $\xi \in \mathfrak{R}_{\nu} \subset \mathfrak{R}$ (for each $\left.\nu\right)$. In particular, if $\xi \in \mathfrak{R}_{\nu}$ and $w \in W$, then $\pi_{\xi, \nu} \cong \pi_{w \xi, w \nu}$.

Although it is not specifically mentioned in [PRV2], it is actually possible to compute the central character of $\pi_{\xi, \nu}$ - and this has a very familiar expression. See Section 4.7

4.2. Constructing the representations $\widehat{\pi}_{\lambda, \nu}$. For his subquotient theorem, Harish-Chandra identified two closed subspaces $\mathfrak{H}_{\xi}^{\prime \prime}(\nu) \subset \mathfrak{H}_{\xi}^{\prime}(\nu) \subset \pi_{\xi, \nu}$, such that the quotient of the larger of them by the smaller one is an irreducible $G$-module. Obtaining a greater understanding of these subquotients of $\pi_{\xi, \nu}$ was one of the main motivations behind [PRV2]; when $G$ is a complex semisimple group, the authors are indeed able to describe these subspaces more easily than Harish-Chandra in the real case. We start with this description. The remainder of this entire section is based on [PRV2, Section 2.4].

Recall from Theorem 4.1 that $\left[\pi_{\xi, \nu}: V_{\overline{\mathfrak{g}}}(\bar{\nu})\right]=1$. Thus, define $\mathfrak{H}_{\xi}^{\prime}(\nu)$ to be the smallest closed $G$-submodule of $\pi_{\xi, \nu}$ containing the unique copy of $V_{\overline{\mathfrak{g}}}(\bar{\nu})$. Inside this, define $\mathfrak{H}_{\xi}^{\prime \prime}(\nu)$ to be the sum of all closed $G$-submodules $M \subset \mathfrak{H}_{\xi}^{\prime}(\nu)$ such that $M \cap V_{\overline{\mathfrak{g}}}(\bar{\nu})=0$. Then $\mathfrak{H}_{\xi}^{\prime \prime}(\nu)$ is a maximal submodule of $\mathfrak{H}_{\xi}^{\prime}(\nu)$, and this leads to the irreducible $G$-representations $\widehat{\pi}_{\lambda, \nu}:=\mathfrak{H}_{\xi}^{\prime}(\nu) / \mathfrak{H}_{\xi}^{\prime \prime}(\nu)$, where $\lambda:=\frac{1}{2}(\xi+\nu)-\rho$ runs over all of $\mathfrak{h}^{*}$ as well. Clearly, $\left[\widehat{\pi}_{\lambda, \nu}: V_{\overline{\mathfrak{g}}}(\mu)\right] \leq \operatorname{dim} V_{\overline{\mathfrak{g}}}(\mu)_{\nu} \forall \mu \in \Lambda^{+}$.

The space $\widehat{\pi}_{\lambda, \nu}$ was shown in PRV2] to have the following properties:

- $\widehat{\pi}_{\lambda, \nu}$ is an irreducible subquotient of $\pi_{\xi, \nu} \subset \mathfrak{H}=L^{2}(K, \mathbb{C}, \mu)$, hence it too is defined on a Hilbert space. Moreover, $\pi_{\xi, \nu}$ is irreducible if and only if $\widehat{\pi}_{\lambda, \nu} \cong \pi_{\xi, \nu}$, if and only if $\left[\widehat{\pi}_{\lambda, \nu}: V_{\overline{\mathfrak{g}}}(\mu)\right]=\operatorname{dim} V_{\overline{\mathfrak{g}}}(\mu)_{\nu}$ for all $\mu \in \Lambda^{+}$.

- $\widehat{\pi}_{\lambda, \nu}$ is an object of $\mathcal{C}(\mathfrak{g} \times \mathfrak{g}, \overline{\mathfrak{g}})$, with minimal type component $\bar{\nu} \in \Lambda^{+} \cap W \nu$. Moreover, $\left[\widehat{\pi}_{\lambda, \nu}: V_{\overline{\mathfrak{g}}}(\bar{\nu})\right]=1$.

- $\widehat{\pi}_{\lambda, \nu}$ has the same infinitesimal character as $\pi_{\xi, \nu}$, where $\lambda=\frac{1}{2}(\xi+\nu)-\rho$.

Note that if $\nu^{\prime} \notin W \nu$, then $\widehat{\pi}_{\lambda^{\prime}, \nu^{\prime}}$ and $\widehat{\pi}_{\lambda, \nu}$ cannot be isomorphic by Equation (2.8), because their minimal types are $\overline{\nu^{\prime}} \neq \bar{\nu}$ respectively.

From above, the modules $\widehat{\pi}_{\lambda, \nu}$ admit infinitesimal characters. It is clear that the highest weight modules $V(\lambda, \mu)$ also admit such characters. Moreover, both of these are families of simple objects in $\mathfrak{C}(\mathfrak{g} \times \mathfrak{g}, \overline{\mathfrak{g}})$. Therefore it is natural to ask if $\widehat{\pi}_{\lambda, \nu}$ is finite-dimensional for some values of the parameters - and if all finite-dimensional simple modules $V(\lambda, \mu)$ are thus covered.

To answer these questions (affirmatively!), Parthasarathy et al studied the "key homomorphisms" $\eta_{V, \mathcal{D}}: \Omega \rightarrow \mathbb{C}$ in greater detail, by relating them to certain homomorphisms $\mathbf{h}^{\Pi}: \Omega \rightarrow$ $P\left(\mathfrak{h}^{*} \times \mathfrak{h}^{*}\right)$. These homomorphisms are the subject of the next subsection.

4.3. Constructing the polynomial-valued maps $\mathbf{h}^{\Pi^{\prime}}$. Recall the "key homomorphism" $\eta_{V, \mathcal{D}}: \Omega \rightarrow \mathbb{C}$, that is defined whenever a simple $\overline{\mathfrak{g}}$-module $\mathcal{D}$ arises with multiplicity one in a simple object $V$ of $\mathcal{C}(\mathfrak{g} \times \mathfrak{g}, \overline{\mathfrak{g}})$. It turns out that there is an explicit construction of the map $\eta_{\widehat{\pi}_{\lambda, \nu}, \bar{\nu}}$ via a different homomorphism $\mathbf{h}^{\Pi^{\prime}}(-; \lambda, \nu)$, which we now present. We explicitly compute both of these maps below in the example of $\mathfrak{g}=\mathfrak{s l}_{2}(\mathbb{C})$, to show that they are equal. This material discusses [PRV2, Sections 2.3, 2.4]. 
To construct the map $\mathbf{h}^{\Pi^{\prime}}$, some more notation is needed. Given $X \in \mathfrak{g}$, define

$$
X_{(1)}:=X \otimes 1, \quad X_{(2)}:=1 \otimes X, \quad \bar{X}:=X_{(1)}+X_{(2)},
$$

and similarly, $\mathfrak{g}_{(1)}, \mathfrak{g}_{(2)} \subset \widehat{\mathfrak{g}}=\mathfrak{g} \oplus \mathfrak{g}$, as well as $\mathfrak{h}_{(1)}$ and so on. Then $X \mapsto \bar{X}$ extends to an isomorphism of associative algebras : $\mathfrak{U} \mathfrak{g} \rightarrow \mathfrak{U} \overline{\mathfrak{g}}$, and similar statements hold for $\mathfrak{g}_{(2)}, \mathfrak{h}(1)$, etc.

Now define $\widehat{\mathfrak{q}}:=\mathfrak{n}_{(1)}^{+} \oplus \mathfrak{n}_{(2)}^{-}$. Note that this is the "positive part" of the triangular decomposition

of $\mathfrak{g} \times \mathfrak{g}$, if we define $\widehat{\Pi}:=\Pi_{(1)} \amalg-\Pi_{(2)}$. This choice of simple roots for $\widehat{\mathfrak{g}}$ comes from the consideration of the conjugation on $\mathfrak{g}$ with respect to a compact form; see a previous footnote. Now $\widehat{\mathfrak{g}}=\overline{\mathfrak{g}} \oplus \mathfrak{h}_{(1)} \oplus \widehat{\mathfrak{q}}$, so by the PBW Theorem,

$$
\mathfrak{U} \widehat{\mathfrak{g}} \cong\left(\mathfrak{U} \overline{\mathfrak{g}} \otimes \mathfrak{U h}_{(1)}\right) \oplus(\mathfrak{U} \mathfrak{\mathfrak { g }}) \widehat{\mathfrak{q}}
$$

as $\mathbb{C}$-vector spaces. Note that every $H \in \operatorname{Sym} \mathfrak{h}$ is a polynomial on $\mathfrak{h}^{*}$ as follows: write $H=$ $p\left(\left\{h_{i}: i \in I\right\}\right)$ for some polynomial $p$. Then $H(\lambda)=p\left(\left\{\lambda\left(h_{i}\right): i \in I\right\}\right)$. This also applies to $H \in \operatorname{Sym}_{\mathfrak{h}_{(1)}}$ or Sym $\overline{\mathfrak{h}}$, for instance, via the obvious isomorphisms mentioned above. Similarly, define $\mathbf{h}^{\mathbf{n}}:=\prod_{i \in I} h_{i}^{n_{i}}$ for $\mathbf{n}=\left(n_{i}\right)_{i \in I} \in \mathbb{Z}_{\geq 0}^{I}$ (which we write as: $\mathbf{n} \geq \mathbf{0}$ ), and also $\overline{\mathbf{h}}^{\mathbf{n}}, \mathbf{h}_{(1)}^{\mathbf{n}}$, and $\lambda(\mathbf{h})^{\mathbf{n}}=\lambda\left(\mathbf{h}^{\mathbf{n}}\right):=\mathbf{h}^{\mathbf{n}}(\lambda)$ from above.

We can now define the homomorphisms in question. Suppose $\omega \in \Omega$, the centralizer of $\overline{\mathfrak{g}}$ in $\mathfrak{U} \widehat{\mathfrak{g}}$. Then there exists a unique $\xi_{\mathbf{n}} \in \mathfrak{U} \mathfrak{g}$ for all $\mathbf{n} \geq \mathbf{0}$, such that

$$
\omega \equiv \sum_{\mathbf{n} \geq \mathbf{0}} \overline{\xi_{\mathbf{n}}} \otimes \mathbf{h}_{(1)}^{\mathbf{n}} \quad \bmod (\mathfrak{U} \widehat{\mathfrak{g}}) \widehat{\mathfrak{q}} .
$$

Since $[\bar{h}, \omega]=0$ for all $h \in \mathfrak{h}$, one checks that $\xi_{\mathbf{n}} \in(\mathfrak{U} \mathfrak{g})_{0}$ for all $\mathbf{n}$. Finally, given any subset $\Pi^{\prime} \subset R$ of simple roots for some Borel subalgebra (equivalently, $\Pi^{\prime}=w \Pi$ for some $w \in W$ ), the maps $\mathbf{h}^{\Pi^{\prime}}$ are defined as follows:

$$
\mathbf{h}^{\Pi^{\prime}}(\omega):=\sum_{\mathbf{n} \geq \mathbf{0}} \beta^{\Pi^{\prime}}\left(\xi_{\mathbf{n}}\right) \otimes \mathbf{h}^{\mathbf{n}} \in \operatorname{Sym}(\mathfrak{h} \times \mathfrak{h}), \quad \mathbf{h}^{\Pi^{\prime}}(\omega ; \lambda, \nu):=\sum_{\mathbf{n} \geq \mathbf{0}} \nu\left(\beta^{\Pi^{\prime}}\left(\xi_{\mathbf{n}}\right)\right) \lambda\left(\mathbf{h}^{\mathbf{n}}\right) \forall \lambda, \nu \in \mathfrak{h}^{*} .
$$

It turns out that these polynomials are very familiar expressions, when $\omega$ is restricted to lie in the center $Z(\mathfrak{U}(\mathfrak{g} \times \mathfrak{g}))$. We see this in Section 4.7 below.

4.4. Relationship between $\widehat{\pi}_{\lambda, \nu}$ and $\mathbf{h}^{\Pi^{\prime}}(-; \lambda, \nu)$. Recall that there are two classes of irreducible admissible representations that are constructed in [PRV2]: the finite-dimensional modules $V(\lambda, \mu)$ for $\lambda, \mu \in \Lambda^{+}$, and the Hilbert space representations $\widehat{\pi}_{\lambda, \nu}$ for $\lambda \in \mathfrak{h}^{*}$ and $\nu \in \Lambda$. In the former case, we define $\nu:=\lambda+w_{\circ} \mu \in \Lambda$ (as in Theorem [3.5); then in both families, the representations all contain the minimal type $V_{\overline{\mathfrak{g}}}(\bar{\nu})$ with multiplicity 1.

Now how does one show that the first of the above families is actually contained inside the second? Similarly, how does one check if two given representations $\widehat{\pi}_{\lambda, \nu}$ and $\widehat{\pi}_{\lambda^{\prime}, \nu^{\prime}}$ are equivalent or not? The answer in both cases is to use the homomorphisms $\mathbf{h}^{\Pi^{\prime}}$, together with Equation (2.8). More precisely, one relates the maps $\mathbf{h}^{\Pi^{\prime}}$ to the homomorphisms $\eta_{\widehat{\pi}_{\lambda, \nu}, \bar{\nu}}$. (Note that this does not completely answer the second question.)

Here are some results from the heart of [PRV2], in which the authors begin to address these questions. The proofs use Theorem 4.1.

Theorem 4.2 ([PRV2]). Suppose $\lambda \in \mathfrak{h}^{*}$ and $w(\nu) \in \Lambda^{+}$for some $\nu \in \Lambda, w \in W$.

(1) Then $\eta_{\widehat{\pi}_{\lambda, \nu}, \bar{\nu}}(-) \equiv \mathbf{h}^{w^{-1} \Pi}(-; \lambda, \nu)$ are homomorphisms $: \Omega \rightarrow \mathbb{C}$.

(2) The maps $\mathbf{h}^{w \Pi}$ are homomorphisms $: \Omega \rightarrow P\left(\mathfrak{h}^{*} \times \mathfrak{h}^{*}\right)$ for all $w \in W$. They are $W$ equivariant in the following sense: for all $\omega \in \Omega, w, w^{\prime} \in W$, and $\lambda, \nu \in \mathfrak{h}^{*}$,

$$
\mathbf{h}^{w^{\prime} w \Pi}\left(\omega ; w^{\prime} * \lambda, w^{\prime} \nu\right)=\mathbf{h}^{w \Pi}(\omega ; \lambda, \nu) .
$$


A consequence of this result is a "first step" towards the classification of the representations $\widehat{\pi}_{\lambda, \nu}$. (This is discussed at greater length in Section 7)

Corollary 4.3. Suppose $(\lambda, \nu),\left(\lambda^{\prime}, \nu^{\prime}\right) \in \mathfrak{h}^{*} \times \Lambda$. Then $\widehat{\pi}_{\lambda, \nu} \cong \widehat{\pi}_{w * \lambda, w \nu}$ for all $w \in W$, while $\widehat{\pi}_{\lambda, \nu}$ and $\widehat{\pi}_{\lambda^{\prime}, \nu^{\prime}}$ are not equivalent if $\nu^{\prime} \notin W \nu$.

Remark 4.4. Another consequence is the following. Note that since every Verma module has a unique simple quotient, hence there exists a unique maximal (left) ideal $\mathfrak{M}_{\lambda} \subset \mathfrak{U} \mathfrak{g}$ containing $\mathfrak{n}^{+}$and ker $\lambda$. If $\lambda \in \Lambda^{+}$, then by Har1,

$$
\mathfrak{M}_{\lambda}=(\mathfrak{U} \mathfrak{g}) \mathfrak{n}^{+}+(\mathfrak{U} \mathfrak{g}) \operatorname{ker} \lambda+\sum_{i \in I} \mathfrak{U} \mathfrak{g} \cdot f_{i}^{\lambda\left(h_{i}\right)+1} .
$$

Thus whenever $w(\nu) \in \Lambda^{+}$for $\nu \in \Lambda$ and $w \in W$, there exists a unique maximal ideal in $\mathfrak{U} \overline{\mathfrak{g}}$ containing ker $\nu \subset \overline{\mathfrak{h}}$ and $\left\{\overline{e_{\alpha}}: \alpha \in w^{-1}\left(R^{+}\right)\right\}$, where $e_{\alpha}$ spans $\mathfrak{g}_{\alpha}$. Call this ideal $\overline{\mathfrak{M}}_{\nu}$. Now since $\widehat{\pi}_{\lambda, \nu}$ is a simple $\mathfrak{g} \times \mathfrak{g}$-module, it is generated by the $\overline{\mathfrak{g}}$-(maximal) weight vector $v_{\bar{\nu}} \in V_{\overline{\mathfrak{g}}}(\bar{\nu})_{\bar{\nu}} \subset \widehat{\pi}_{\lambda, \nu}$. Moreover, $\Omega$ acts on $V_{\overline{\mathfrak{g}}}(\bar{\nu})$ by $\mathbf{h}^{w^{-1} \Pi}(-; \lambda, \nu)$. By Equation (2.8), this data uniquely determines $\widehat{\pi}_{\lambda, \nu}$ up to isomorphism. This implies the following result from [PRV1]:

There exists a unique maximal ideal $\mathfrak{M}_{\lambda, \nu} \subset \mathfrak{U}(\mathfrak{g} \times \mathfrak{g})$ containing $\operatorname{ker} \mathbf{h}^{w^{-1} \Pi}(-; \lambda, \nu) \subset \Omega$ and $\overline{\mathfrak{M}}_{\nu}$. Moreover, $\widehat{\pi}_{\lambda, \nu} \cong \mathfrak{U}(\mathfrak{g} \times \mathfrak{g}) / \mathfrak{M}_{\lambda, \nu}$.

Note that $\mathbf{h}^{\Pi^{\prime}}: \Omega \rightarrow P\left(\mathfrak{h}^{*} \times \mathfrak{h}^{*}\right)$ is a homomorphism for each $\Pi^{\prime}=w \Pi$. Thus, $\mathbf{h}^{\Pi^{\prime}}(-; \lambda, \nu)$ is a homomorphism : $\Omega \rightarrow \mathbb{C}$ for all $\lambda, \nu \in \mathfrak{h}^{*}$. The strategy in PRV2 for showing this is to restrict to the Zariski dense subset of $(\lambda, \nu)$ arising from finite-dimensional modules. The authors prove that if $\mu$ and $\Pi^{\prime}$ are chosen "suitably", then $\mathbf{h}^{\Pi^{\prime}}(-; \lambda, \nu) \equiv \eta_{V(\lambda, \mu), \bar{\nu}}(-)$ is a homomorphism $: \Omega \rightarrow \mathbb{C}$. Hence so is $\mathbf{h}^{\Pi^{\prime}}$ at all values of $(\lambda, \nu)$. This analysis leads to the next topic.

4.5. Relationship between $V(\lambda, \mu)$ and $\mathbf{h}^{\Pi^{\prime}}(-; \lambda, \nu)$. Consider the other family of simple $\mathfrak{C}(\mathfrak{g} \times \mathfrak{g}, \overline{\mathfrak{g}})$-modules studied in [PRV2]: the finite-dimensional $V(\lambda, \mu)$. From above, the minimal type of such a module is $\overline{\lambda+w_{\circ} \mu}$. Now consider the "converse" question: given $\lambda \in \mathfrak{h}^{*}$ and $\nu \in \Lambda$, is it possible to produce $\mu \in \Lambda^{+}$such that $V(\lambda, \mu)$ has minimal type $\bar{\nu}$ ? Supporting evidence for such a claim is given by the following result, in light of Equation (2.8).

Theorem 4.5 ([PRV2] $)$. Suppose $\lambda \in \Lambda^{+}$and $\nu \in \lambda-\Lambda^{+}$. Choose $w \in W$ such that $w(\nu) \in \Lambda^{+}$ and define $\mu:=-w_{\circ}(\lambda-\nu) \in \Lambda^{+}$. Then $V(\lambda, \mu)$ has minimal type $V_{\overline{\mathfrak{g}}}(\bar{\nu})$; moreover,

$$
\eta_{V(\lambda, \mu), \bar{\nu}}(-) \equiv \mathbf{h}^{w^{-1} \Pi}(-; \lambda, \nu)
$$

Restating this allows us to answer the "converse" question, which can also be found in [Du2].

Corollary 4.6. For all $\lambda, \mu \in \Lambda^{+}, V(\lambda, \mu) \cong \widehat{\pi}_{\lambda, \lambda+w_{\circ} \mu}$.

Proof. Note that $\nu=\lambda+w_{\circ} \mu \in \lambda-\Lambda^{+} \Leftrightarrow \mu=-w_{\circ}(\lambda-\nu) \in \Lambda^{+}$. Thus, set $\nu:=\lambda+w_{\circ} \mu$ and choose $w \in W$ such that $w(\nu) \in \Lambda^{+}$. Then using Theorems 4.2 and 4.5,

$$
\eta_{\widehat{\pi}_{\lambda, \lambda+w_{\circ} \mu}, \bar{\nu}}(-) \equiv \mathbf{h}^{w^{-1} \Pi}\left(-; \lambda, \lambda+w_{\circ} \mu\right) \equiv \eta_{V(\lambda, \mu), \bar{\nu}}(-)
$$

on $\Omega$. Moreover, $\bar{\nu}$ is the (multiplicity one) minimal type component of both irreducible modules, by the PRV Theorem. The result follows by applying Equation (2.8).

Remark 4.7. A word of caution: note that $V(\lambda) \otimes V(\mu) \cong V(\mu) \otimes V(\lambda)$ as $\overline{\mathfrak{g}}$-modules for $\lambda, \mu \in \mathfrak{h}^{*}$, since $\mathfrak{U} \mathfrak{g}$ is cocommutative. Thus, their minimal types are also equal (when $\lambda, \mu \in$ $\left.\Lambda^{+}\right): \overline{\lambda+w_{\circ} \mu}=\overline{\mu+w_{\circ} \lambda}$. This implies that the action of $Z(\mathfrak{U} \overline{\mathfrak{g}})$ is equal on both modules. However, $V(\lambda, \mu)$ and $V(\mu, \lambda)$ are non-isomorphic simple $\mathfrak{g} \times \mathfrak{g}$-modules if $\lambda \neq \mu$. Similarly, the infinitesimal characters are not equal on all of $Z(\mathfrak{U}(\mathfrak{g} \times \mathfrak{g}))$ - and hence the key homomorphisms $\eta_{V(\lambda, \mu), \bar{\nu}}, \eta_{V(\mu, \lambda), \bar{\nu}}$ do not agree on all of $\Omega$ - unless $\lambda=\mu$. 
4.6. Example: the case of $\mathfrak{s l}_{2}(\mathbb{C})$. We now verify a part of Theorem 4.5 in the special case of $\mathfrak{g}=\mathfrak{s l}_{2}(\mathbb{C})$, in which case it is not hard to compute both the homomorphisms in question - at least, on a particular finitely generated subalgebra $\Omega^{\prime} \subset \Omega$.

For convenience, denote the two factors in $\widehat{\mathfrak{g}}$ as $\mathfrak{g}_{k}$ for $k=1,2$ (as opposed to $\mathfrak{g}_{(1)}$ and $\left.\mathfrak{g}_{(2)}\right)$, with bases $\left\{e_{k}, f_{k}, h_{k}\right\}$. Now for all $0<n_{2} \leq n_{1} \in \mathbb{N}$, the "tensor product" module $V_{1}\left(n_{1}\right) \otimes V_{2}\left(n_{2}\right)$ is a simple object in $\mathcal{C}(\mathfrak{g} \times \mathfrak{g}, \overline{\mathfrak{g}})$, of highest weight $\left(n_{1}, n_{2}\right)$. Restricted to $\overline{\mathfrak{g}}$, it decomposes according to the Clebsch-Gordan coefficients:

$$
V\left(n_{1}, n_{2}\right) \cong_{\overline{\mathfrak{g}}} V\left(n_{1}\right) \otimes V\left(n_{2}\right) \cong V_{\overline{\mathfrak{g}}}\left(n_{1}+n_{2}\right) \oplus V_{\overline{\mathfrak{g}}}\left(n_{1}+n_{2}-2\right) \oplus \cdots \oplus V_{\overline{\mathfrak{g}}}\left(n_{1}-n_{2}\right),
$$

where $V_{\overline{\mathfrak{g}}}(n)$ is a finite-dimensional irreducible $\overline{\mathfrak{g}}$-module of highest weight $n$ (equivalently, of dimension $n+1)$. Now denote the highest weight generators of $V_{i}\left(n_{i}\right)$ by $v_{n_{1}}$ and $v_{n_{2}}^{\prime}$ respectively, and define the weight basis $v_{n_{1}-2 i}:=\left(f^{i} / i !\right) v_{n_{1}}$ of $V_{1}\left(n_{1}\right)$, with $0 \leq i<\operatorname{dim} V_{1}\left(n_{1}\right)$. Similarly define $v_{n_{2}-2 j}^{\prime} \in V_{2}\left(n_{2}\right)$. One checks using Equation (1.1) that

$$
v_{n_{1}-n_{2}}^{\prime \prime}:=\sum_{j=0}^{n_{2}}(-1)^{j} j ! \cdot n_{1}\left(n_{1}-1\right) \cdots\left(n_{1}-j+1\right) \cdot v_{n_{1}-2 j} \otimes v_{2 j-n_{2}}^{\prime}
$$

is a weight vector in $V\left(n_{1}, n_{2}\right)$ which is killed by $e_{1}+e_{2}:=e_{1} \otimes 1+1 \otimes e_{2}$. Hence it generates the minimal type (i.e., the PRV component) $V_{\overline{\mathfrak{g}}}\left(n_{1}-n_{2}\right)$, and we have:

$$
\lambda=n_{1}, \quad \mu=n_{2}, \quad \nu=\lambda+w_{\circ} \mu=n_{1}-n_{2} \geq 0, \quad \bar{\nu}=\nu \in \Lambda^{+} .
$$

Note that the subalgebra $\Omega$ that commutes with $\overline{\mathfrak{g}}$ in $\mathfrak{U}(\widehat{\mathfrak{g}})$ contains the center of $\mathfrak{U}(\widehat{\mathfrak{g}})$. This is freely generated by the two Casimir operators

$$
\Delta_{i}:=4 f_{i} e_{i}+h_{i}^{2}+2 h_{i}=4 e_{i} f_{i}+h_{i}^{2}-2 h_{i} .
$$

Clearly, $\Delta_{1} \otimes 1$ acts on $V_{1}\left(n_{1}\right) \otimes V_{2}\left(n_{2}\right)$ by the scalar $n_{1}^{2}+2 n_{1}$; similarly, $1 \otimes \Delta_{2}$ acts by $n_{2}^{2}+2 n_{2}$. Moreover, $\bar{\Delta}=4\left(f_{1}+f_{2}\right)\left(e_{1}+e_{2}\right)+\left(h_{1}+h_{2}\right)^{2}+2\left(h_{1}+h_{2}\right)$ lies in $\Omega$ as well; by $\mathfrak{s l}_{2}$-theory, it acts on $v_{n_{1}-n_{2}}^{\prime \prime}$ via: $\bar{\Delta} \cdot v_{n_{1}-n_{2}}^{\prime \prime}=\left(\left(n_{1}-n_{2}\right)^{2}+2\left(n_{1}-n_{2}\right)\right) v_{n_{1}-n_{2}}^{\prime \prime}$. Since $\bar{\Delta}$ commutes with $\overline{\mathfrak{g}}$, it acts on $V_{\overline{\mathfrak{g}}}\left(n_{1}-n_{2}\right)$ by this same scalar. This allows us to determine $\eta_{V\left(n_{1}, n_{2}\right), n_{1}-n_{2}}$ - at least, on the subalgebra $\Omega^{\prime}:=\mathbb{C}\left[\Delta_{1}, \Delta_{2}, \bar{\Delta}\right] \subset \Omega$ :

$\eta_{V\left(n_{1}, n_{2}\right), n_{1}-n_{2}}\left(\Delta_{i}\right):=n_{i}^{2}+2 n_{i}=\chi\left(n_{i}\right)(\Delta)(i=1,2), \quad \eta_{V\left(n_{1}, n_{2}\right), n_{1}-n_{2}}(\bar{\Delta}):=\chi\left(n_{1}-n_{2}\right)(\Delta)$.

Now consider $\mathbf{h}^{\Pi}(-; \lambda, \nu)$. Note from above that $\bar{\nu}=\nu=\lambda+w_{\circ} \mu=n_{1}-n_{2}$, so $w=1$. Since $\widehat{\mathfrak{q}}$ is spanned by $e_{1}$ and $f_{2}$, projecting the various $\Delta_{i}$ onto $\mathfrak{U} \overline{\mathfrak{g}} \otimes \mathfrak{U} \mathfrak{h}_{(1)}$ modulo $\widehat{\mathfrak{q}}$ yields

$$
\Delta_{1}=4 f_{1} e_{1}+h_{1}^{2}+2 h_{1} \equiv h_{1}^{2}+2 h_{1} \quad \bmod (\mathfrak{U} \widehat{\mathfrak{g}}) \widehat{\mathfrak{q}} .
$$

Hence $\mathbf{h}^{\Pi}\left(\Delta_{1} ; \lambda, \nu\right)=\lambda\left(h_{1}\right)^{2}+2 \lambda\left(h_{1}\right)=n_{1}^{2}+2 n_{1}$, as in Equation (4.8). Similarly,

$$
\begin{aligned}
\Delta_{2} & =4 e_{2} f_{2}+h_{2}^{2}-2 h_{2} \equiv h_{2}^{2}-2 h_{2} \equiv\left(h_{1}+h_{2}\right)^{2}-2\left(h_{1}+h_{2}\right)-h_{1}^{2}-2 h_{1} h_{2}+2 h_{1} \\
& \equiv \bar{h}^{2}-2 \bar{h}-2 \bar{h} \otimes h_{1}+h_{1}^{2}+2 h_{1} \quad \bmod (\mathfrak{U} \widehat{\mathfrak{g}}) \widehat{\mathfrak{q}} .
\end{aligned}
$$

Computing $\mathbf{h}^{\Pi}\left(\Delta_{2} ; \lambda, \nu\right)$ amounts to evaluating this polynomial at $\left(\nu(\bar{h}), \lambda\left(h_{1}\right)\right)=\left(n_{1}-n_{2}, n_{1}\right)$. This yields $n_{2}^{2}+2 n_{2}$, as desired. Finally, $\bar{\Delta}=2 \bar{f} \bar{e}+\bar{h}^{2}+2 \bar{h}$. To evaluate $\mathbf{h}^{\Pi}(\bar{\Delta} ; \lambda, \nu)$, we must first apply the Harish-Chandra projection $\beta^{\Pi}$ to this expression, which kills the first term. Now evaluating at $\left(\bar{h}, h_{1}\right)=\left(n_{1}-n_{2}, n_{1}\right)$, we obtain $\chi\left(n_{1}-n_{2}\right)(\Delta)$, as in Equation (4.8). Thus, $\mathbf{h}^{\Pi}\left(\Delta^{\prime} ; n_{1}, n_{1}-n_{2}\right) \equiv \eta_{V\left(n_{1}, n_{2}\right), n_{1}-n_{2}}\left(\Delta^{\prime}\right)$ for $\Delta^{\prime}=\Delta_{1}, \Delta_{2}, \bar{\Delta}$; assuming that both of these are homomorphisms implies equality on all of $\Omega^{\prime}$. 
4.7. Infinitesimal characters. Although it does not seem to be explicitly mentioned in [PRV2], the above facts allow us to compute the infinitesimal characters of the representations $\pi_{\xi, \nu}$ - or equivalently, of $\widehat{\pi}_{\lambda, \nu}$. In fact, we prove a stronger result.

Theorem 4.9. For all $\lambda, \nu \in \mathfrak{h}^{*}, w \in W$, and $z \in Z(\mathfrak{U}(\mathfrak{g} \times \mathfrak{g}))$,

$$
\mathbf{h}^{w \Pi}(z ; \lambda, \nu)=\chi(\lambda, \nu-\lambda-2 \rho)(z),
$$

where $\chi\left(\lambda, \lambda^{\prime}\right)$ is the central character for the $\mathfrak{g} \times \mathfrak{g}$-Verma module $M\left(\lambda, \lambda^{\prime}\right)$. Now given $\xi \in \mathfrak{h}^{*}$ and $\nu \in \Lambda$, define $\lambda:=\frac{1}{2}(\xi+\nu)-\rho$. Then $\chi_{\pi_{\xi, \nu}}=\chi_{\widehat{\pi}_{\lambda, \nu}}=\chi(\lambda, \nu-\lambda-2 \rho)$.5 Moreover, $\chi_{\pi_{w \xi, w \nu}}=\chi_{\widehat{\pi}_{w * \lambda, w \nu}}=\chi(w * \lambda, w *(\nu-\lambda-2 \rho))=\chi(\lambda, \nu-\lambda-2 \rho)$.

For example, consider the situation where $\widehat{\pi}_{\lambda, \nu}$ is finite-dimensional. Thus, $\nu \in \lambda-\Lambda^{+}$, and $\widehat{\pi}_{\lambda, \nu}=V(\lambda, \mu)$, where $\mu:=-w_{\circ}(\lambda-\nu) \in \Lambda^{+}$. Thus, $\nu=\lambda+w_{\circ} \mu$. In this case, the result says that the second component of the central character is:

$$
\chi(\nu-\lambda-2 \rho)=\chi\left(\lambda+w_{\circ} \mu-\lambda-2 \rho\right)=\chi\left(w_{\circ} \mu+w_{\circ} \rho-\rho\right)=\chi\left(w_{\circ} * \mu\right),
$$

since $w_{\circ} \rho=-\rho$. Hence $\chi_{V(\lambda, \mu)}=\chi\left(\lambda, w_{\circ} * \mu\right)=\chi(\lambda, \mu)$ (by Theorem 1.2), as expected.

Proof. We use a "Zariski density" argument as in [PRV2], that Varadarajan attributes to HarishChandra in [Va]. By Harish-Chandra's Theorem 1.2, every central character of $Z(\mathfrak{U}(\mathfrak{g} \times \mathfrak{g}))$ is of the form $\chi\left(\mu_{1}, \mu_{2}\right)$ for some $\mu_{i} \in \mathfrak{h}^{*}$. Moreover, $Z(\mathfrak{U}(\mathfrak{g} \times \mathfrak{g})) \subset \Omega$, so we can evaluate $\mathbf{h}^{w^{-1} \Pi}(-; \lambda, \nu)$ on it. For all $z \in Z(\mathfrak{U} \mathfrak{g})$, one uses the definitions to check that $\mathbf{h}^{w^{-1} \Pi}\left(z_{(1)} ; \lambda, \nu\right)=$ $(\nu \otimes \lambda)\left(1 \otimes \beta^{\Pi}\left(z_{(1)}\right)\right)=\chi(\lambda)(z)$ for all $\lambda, \nu \in \mathfrak{h}^{*}$ and all $\Pi^{\prime}$.

For $z_{(2)}$ in the second copy of the center, first suppose that $\nu \in \Lambda^{+}$. Write the Harish-Chandra projection of $z$, but now using the decomposition $\mathfrak{U} \mathfrak{g}=\mathfrak{U} \mathfrak{n}^{+} \otimes \mathfrak{U} \mathfrak{h} \otimes \mathfrak{U} \mathfrak{n}^{-}$. In other words, compute $\beta^{w_{\circ} \Pi}(z)$, since $z_{(2)} \equiv\left(\beta^{w_{\circ} \Pi}(z)\right)_{(2)} \bmod \left(\mathfrak{U}(\widehat{\mathfrak{g}}) \widehat{\mathfrak{q}}\right.$. Now use the basis $h_{i(2)}$ of $\mathfrak{h}_{(2)}$ to write out the above as a polynomial:

$$
\left(\beta^{w_{\circ} \Pi}(z)\right)_{(2)}=p\left(\left\{h_{i(2)}: i \in I\right\}\right)=p\left(\left\{\overline{h_{i}}-h_{i(1)}: i \in I\right\}\right) .
$$

Hence $\mathbf{h}^{\Pi}\left(z_{(2)} ; \lambda, \nu\right)$ involves acting by $\nu$ on $\overline{h_{i}}$ and by $\lambda$ on $h_{i(1)}$. Now recall that for all $z \in Z(\mathfrak{U} \mathfrak{g}), \bar{z} \in Z(\mathfrak{U} \overline{\mathfrak{g}}) \subset \Omega$. Using these facts, and fixing $\nu^{\prime} \in \mathfrak{h}^{*}$, compute using Theorem 4.2.

$$
\begin{aligned}
\mathbf{h}^{\Pi}\left(z_{(2)} ; \lambda, \nu\right) & =p\left(\left\{\nu\left(h_{i}\right)-\lambda\left(h_{i}\right)\right\}\right)=p\left(\left\{(\nu-\lambda)\left(h_{i}\right)\right\}\right)=(\nu-\lambda)\left(\beta^{w_{\circ} \Pi}(z)\right) \nu^{\prime}(1) \\
& =\mathbf{h}^{w_{\circ} \Pi}\left(\bar{z} ; \nu^{\prime}, \nu-\lambda\right)=\mathbf{h}^{\Pi}\left(\bar{z} ; w_{\circ} * \nu^{\prime}, w_{\circ}(\nu-\lambda)\right)=\chi\left(w_{\circ}(\nu-\lambda)\right)(z),
\end{aligned}
$$

since $w_{\circ}=w_{\circ}^{-1}$. By Theorem 1.2, twist this weight by $w_{\circ}$; thus for all $(z, \lambda, \nu) \in Z(\mathfrak{U} \mathfrak{g}) \times \mathfrak{h}^{*} \times \Lambda^{+}$,

$$
\mathbf{h}^{\Pi}\left(z_{(2)} ; \lambda, \nu\right)=\chi\left(w_{\circ} * w_{\circ}(\nu-\lambda)\right)(z)=\chi(\nu-\lambda-2 \rho)(z) .
$$

Now fix any weight $\lambda$ and any central $z$, and consider the map $h_{z, \lambda}: \mathfrak{h}^{*} \rightarrow \mathbb{C}$, given by:

$$
h_{z, \lambda}(\nu):=\mathbf{h}^{\Pi}\left(z_{(2)} ; \lambda, \nu\right)-\chi(\nu-\lambda-2 \rho)(z) .
$$

It is clear that $h_{z, \lambda}$ is a polynomial map, which vanishes if $\nu$ lies in the Zariski dense subset $\Lambda^{+} \subset \mathfrak{h}^{*}$. But then $h_{z, \lambda} \equiv 0$ as polynomials. (This is the aforementioned Zariski density argument; it is analogous to saying that if a polynomial $p\left(T_{1}, \ldots, T_{n}\right): \mathbb{C}^{n} \rightarrow \mathbb{C}$ is identically zero on $z_{0}+\mathbb{Z}_{>0}^{n}$ for some $z_{0} \in \mathbb{C}^{n}$, then $p \equiv 0$.) We conclude that for all $\lambda, \nu \in \mathfrak{h}^{*}$ and $z \in Z(\mathfrak{U} \mathfrak{g})$, $\mathbf{h}^{\Pi}(z ; \lambda, \nu)=\chi(\lambda, \nu-\lambda-2 \rho)(z)$. It is also easy to check that the following holds:

$$
w *(\nu-\lambda-2 \rho)=w \nu-w * \lambda-2 \rho \forall w \in W, \nu, \lambda \in \mathfrak{h}^{*} .
$$

\footnotetext{
${ }^{5}$ This assertion about central characters of Harish-Chandra modules can be found in Du2, for instance.
} 
Now given any $w \in W$, compute using Theorems 1.2 and 4.2 .

$$
\begin{aligned}
\mathbf{h}^{w^{-1} \Pi}(z ; \lambda, \nu) & =\mathbf{h}^{\Pi}(z ; w * \lambda, w \nu)=\chi(w * \lambda, w \nu-w * \lambda-2 \rho)(z) \\
& =\chi(w * \lambda, w *(\nu-\lambda-2 \rho))(z)=\chi(\lambda, \nu-\lambda-2 \rho)(z) .
\end{aligned}
$$

This proves the main assertion of the theorem; the rest follow easily. For instance, if $w \nu \in \Lambda^{+}$, then considering the action of any central $z$ on the minimal type (and using Theorem 4.2),

$$
\chi_{\widehat{\pi}_{\lambda, \nu}}(z)=\eta_{\widehat{\pi}_{\lambda, \nu}, \bar{\nu}}(z)=\mathbf{h}^{w^{-1} \Pi}(z ; \lambda, \nu)=\chi(\lambda, \nu-\lambda-2 \rho)(z) .
$$

Remark 4.11. A similar result follows from the definitions: given $z \in Z(\mathfrak{U} \mathfrak{g}), \lambda, \nu \in \mathfrak{h}^{*}$, and $w \in W, \mathbf{h}^{w^{-1} \Pi}(\bar{z} ; \lambda, \nu)=\chi(w(\nu))(z)$. Note that this was shown in [PRV2] only when $w(\nu) \in \Lambda^{+}$. Now since the action of any $w \in W$ on $\mathfrak{h}^{*}$ is a linear - hence, polynomial - map, once again a Zariski density argument can be used to extend the result to all $\nu \in \mathfrak{h}^{*}$, for any fixed $z \in Z(\mathfrak{U} \mathfrak{g})$.

In the above result, observe that the above recipe for the central character is " $W$-equivariant", in that the last equation in the statement of the theorem (which is basically Equation (4.10) ) holds. One can check that this is not always so: in other words, if we use $\chi(\lambda, w *(\nu-\lambda-2 \rho))$ to denote the central character (via Theorem 1.2) for arbitrary $w \neq 1 \in W$ - such as $w=w_{\circ}$, say. However, one can check that $W$-equivariance does hold if $w$ is central in $W$.

4.8. Remarks. We conclude this section with some remarks. Note that the presentation in PRV2] of the material in this section is motivated by the approach of Harish-Chandra. Thus, it differs somewhat from the presentation in this article.

More precisely, the philosophy in [PRV2] (as per the historical account given in [Va]) was to use Harish-Chandra's density theorem, which roughly says that among all irreducible $G$-modules containing a given irreducible finite-dimensional $\mathfrak{k}$-module $\mathcal{D}$, the ones that are finite-dimensional form a Zariski dense set. What this means is that the homomorphisms $\mathbf{h}^{\Pi^{\prime}}(-; \lambda, \nu)$ correspond to finite-dimensional representations at special lattice points, as in Corollary [4.6 - and the set of these lattice points is Zariski dense in $\mathfrak{h}^{*} \times \mathfrak{h}^{*}$. If we replace these by more general points, then the homomorphisms in question correspond to infinite-dimensional representations.

In view of this perspective, the approach in PRV2 was to "alternate" between the representations and the homomorphisms. Here is their strategy:

- Identify and study the minimal type in all finite-dimensional simple $\widehat{\mathfrak{g}}$-modules $V(\lambda, \mu)$.

- Explicitly compute the polynomials $\mathbf{h}^{\Pi^{\prime}}$ for these modules, and prove that $\eta_{V(\lambda, \mu), \bar{\nu}}(-) \equiv$ $\mathbf{h}^{w^{-1} \Pi}(-; \lambda, \nu)$, where $\nu=\lambda+w_{\circ} \mu$ and $\bar{\nu}=w(\nu) \in \Lambda^{+}$.

- Motivated by this, claim that there are simple modules $\widehat{\pi}_{\lambda, \nu}$ in $\mathcal{C}(\mathfrak{g} \times \mathfrak{g}, \overline{\mathfrak{g}})$ for all $\lambda \in \mathfrak{h}^{*}$ and $\nu \in \Lambda$, with minimal type $\bar{\nu}$; now construct these.

- Finally, prove that $\eta_{\widehat{\pi}_{\lambda, \nu}, \bar{\nu}}(-)$ indeed equals $\mathbf{h}^{w^{-1} \Pi}(-; \lambda, \nu)$ for these modules.

\section{The Rings $\mathcal{R}_{\nu, \Pi^{\prime}}$ And the PRV Determinants}

The next object of study in [PRV2] is the image $\mathcal{R}_{\nu, w^{-1} \Pi}$ of the map $\mathbf{h}^{w^{-1} \Pi}(-;-, \nu): \Omega \rightarrow$ Sym $\mathfrak{h}$, where $w(\nu) \in \Lambda^{+}$. This was done in detail in [PRV2, Section 3] using deep results of Kostant [Ko2, such as his separation of variables theorem (2.4). These results lead to the definition and study of the so-called PRV determinants, which we will discuss below. 
5.1. The rings $\mathcal{R}_{\nu, \Pi^{\prime}}$. To state the next result, we need some notation. Recall the stabilizer subgroup $W_{\nu}$ of a weight $\nu$, as well as the twisted action of the Weyl group $*$ on $\mathfrak{h}^{*}$, which transfers to $\mathfrak{h}$ and then extends to $\operatorname{Sym} \mathfrak{h}=P\left(\mathfrak{h}^{*}\right)$.

Theorem 5.1 ([RV2]). For all $\nu \in \Lambda$ and $w \in W$ such that $w(\nu) \in \Lambda^{+}, \mathcal{R}_{\nu, w^{-1} \Pi} \subset \mathbf{I}_{\nu}$, where

$$
\mathbf{I}_{\nu}:=\left\{p \in P\left(\mathfrak{h}^{*}\right): w * p=p \forall w \in W_{\nu}\right\} .
$$

If $\nu=0$ or $\nu\left(h_{i}\right)>0$ for all $i \in I$, then $\mathcal{R}_{\nu, \Pi}=\mathbf{I}_{\nu}$. For general $\nu \in \Lambda^{+}$, define

$$
(\mathfrak{U} \mathfrak{g})_{0}(\nu):=\left\{a \in(\mathfrak{U} \mathfrak{g})_{0}:\left(\operatorname{ad} e_{i}\right)^{\nu\left(h_{i}\right)+1}(a)=0 \forall i \in I\right\} .
$$

Then $\mathcal{R}_{\nu, \Pi}=w_{\circ} * \beta^{\Pi}\left((\mathfrak{U} \mathfrak{g})_{0}\left(-w_{\circ} \nu\right)\right)$, and $\mathcal{R}_{w \nu, w \Pi}=w * \mathcal{R}_{\nu, \Pi}$ for all $w \in W$.

Note that if $\nu\left(h_{i}\right)>0$ for all $i$ (i.e., $\nu$ is regular), then $\mathbf{I}_{\nu}=P\left(\mathfrak{h}^{*}\right)$. Moreover, $\mathbf{I}_{0}=P\left(\mathfrak{h}^{*}\right)^{(W, *)}$.

Remark 5.2. The authors claim in $[\mathrm{PRV} 2]$ that they have proved that $\mathcal{R}_{\nu, \Pi}=\mathbf{I}_{\nu}$ for all $\nu \in \Lambda^{+}$, using a case-by-case analysis.

We also remark that Theorem 5.1 has the following consequence for $\nu=0$ or regular $\nu$.

Corollary 5.3 ([PRV2] $)$. Suppose $\nu\left(h_{i}\right)>0$ for all $i$, and $\lambda, \lambda^{\prime} \in \mathfrak{h}^{*}$. Then,

$$
\widehat{\pi}_{\lambda, 0} \cong \widehat{\pi}_{\lambda^{\prime}, 0} \Longleftrightarrow \lambda^{\prime} \in W * \lambda,
$$

whereas the representations $\widehat{\pi}_{\lambda, \nu}$ are inequivalent for all $\lambda$.

The proofs of these results are carefully developed in [PRV2, Section 3], via many intermediate lemmas. These lemmas heavily use results developed by Kostant in Ko2, which deal with the symmetrization map and with finite-dimensional $\mathfrak{g}$-submodules of Sym $\mathfrak{g}$ and $\mathfrak{U} \mathfrak{g}$. In the next part, we discuss some of these results, and show how they can be used to define and study the "PRV determinants".

We end this part by discussing the case of $\mathfrak{s l}_{2}$, where we classify all of the modules $\widehat{\pi}_{\lambda, \nu}$.

Example 5.4. Suppose $\mathfrak{g}=\mathfrak{s l}_{2}(\mathbb{C})$. Using the results of Section 4.6, compute with $\nu \in \mathbb{Z}_{\geq 0}$ :

$\mathbf{h}^{\Pi}\left(\Delta_{1} ;-, \nu\right)=h_{1}^{2}+2 h_{1}, \quad \mathbf{h}^{\Pi}\left(\Delta_{2} ;-, \nu\right)=\nu^{2}-2 \nu-2 \nu h_{1}+h_{1}^{2}+2 h_{1}, \quad \mathbf{h}^{\Pi}(\bar{\Delta} ;-, \nu)=\nu^{2}+2 \nu$.

It is now clear that if $\nu=0$, then these generate $\mathbb{C}\left[h_{1}^{2}+2 h_{1}\right]=\mathbb{C}\left[h_{1}\right]^{(W, *)}$. The above result says that $\mathbf{h}^{\Pi}(\omega ;-, \nu)$ is an element of this ring for all $\omega \in \Omega$. Similarly, if $\nu>0$ (abusing notation), then the above polynomials already generate all of $\mathbb{C}\left[h_{1}\right]$ as desired.

Also note that we can classify (all equivalences between) the representations $\widehat{\pi}_{\lambda, \nu}$ : given $(\lambda, \nu) \neq\left(\lambda^{\prime}, \nu^{\prime}\right)$ in $\mathfrak{h}^{*} \times \Lambda$, we claim:

$$
\widehat{\pi}_{\lambda, \nu} \cong \widehat{\pi}_{\lambda^{\prime}, \nu^{\prime}} \Longleftrightarrow\left(\lambda^{\prime}, \nu^{\prime}\right)=(-\lambda-2,-\nu) .
$$

The backward implication follows from Corollary 4.3, and conversely, $\nu^{\prime}= \pm \nu$. Then the calculations above imply that $\mathbf{h}^{\Pi}\left(\Delta_{1} ; \lambda, \nu\right)=\mathbf{h}^{\Pi}\left(\Delta_{1} ; \lambda^{\prime}, \nu^{\prime}\right)$, whence $\lambda^{\prime}=\lambda,-\lambda-2$. This shows the claim when $\nu^{\prime}= \pm \nu=0$. Otherwise we may assume that $\nu^{\prime}=\nu>0$ (using the backward implication). Now evaluate $\mathbf{h}^{\Pi}\left(\bar{\Delta}+\Delta_{1}-\Delta_{2} ;-, \nu\right)$ at $\lambda, \lambda^{\prime}$. Then $\nu \neq 0 \Longrightarrow \lambda=\lambda^{\prime}$.

These calculations naturally lead to the question of classifying the representations $\widehat{\pi}_{\lambda, \nu}$ for general semisimple $\mathfrak{g}$ - and more generally, the classification of all simple objects of $\mathcal{C}(\mathfrak{g} \times \mathfrak{g}, \overline{\mathfrak{g}})$. (Recall Corollary 4.3.) These questions will be discussed in the concluding section of this article. 
5.2. Kostant's separation of variables. In order to discuss PRV determinants, we need some preliminaries. Recall the symmetrization map $\boldsymbol{\lambda}$ from [Har2], which is the unique linear isomorphism : Sym $\mathfrak{g} \rightarrow \mathfrak{U} \mathfrak{g}$ satisfying: $\boldsymbol{\lambda}(1)=1$ and for all $r>0$ and $X_{1}, \ldots, X_{r} \in \mathfrak{g}$,

$$
\lambda\left(X_{1} \ldots X_{r}\right)=\frac{1}{r !} \sum_{\sigma \in S_{r}} X_{\sigma(1)} \ldots X_{\sigma(r)} .
$$

Also recall that the adjoint action of $\mathfrak{g}$ on itself can be uniquely extended to derivations $\Phi$ : $\mathfrak{g} \rightarrow$ Sym $\mathfrak{g}$ and $\Theta: \mathfrak{g} \rightarrow \mathfrak{U} \mathfrak{g}$. Thus, both of these algebras are $\mathfrak{g}$-modules, and $\boldsymbol{\lambda}$ is a $\mathfrak{g}$-module isomorphism: $\boldsymbol{\lambda} \circ \Phi(X)=\Theta(X) \circ \boldsymbol{\lambda}$ for all $X \in \mathfrak{g}$. Moreover, the centers are isomorphic via $\boldsymbol{\lambda}$. Namely, $\boldsymbol{\lambda}:(\operatorname{Sym} \mathfrak{g})^{G_{0}} \rightarrow Z(\mathfrak{U} \mathfrak{g})=(\mathfrak{U} \mathfrak{g})^{G_{0}}$, where $G_{0}$ is the adjoint group of $\mathfrak{g}$.

Now given $\mu \in \Lambda^{+}$, define the following copies of the $\mathfrak{g}$-module $V(\mu)$ in Sym $\mathfrak{g}$ and $\mathfrak{U} \mathfrak{g}$ :

$$
\mathcal{L}^{\mu}:=\operatorname{Hom}_{\mathfrak{g}}(V(\mu), \operatorname{Sym} \mathfrak{g}), \quad \mathcal{L}^{\mu}(\mathfrak{U} \mathfrak{g}):=\operatorname{Hom}_{\mathfrak{g}}(V(\mu), \mathfrak{U} \mathfrak{g})=\left\{\boldsymbol{\lambda} \circ M: M \in \mathcal{L}^{\mu}\right\} .
$$

In [Ko2], Kostant showed that these are both free modules of rank $d_{\mu}:=\operatorname{dim} V(\mu)_{0}$, over $(\operatorname{Sym} \mathfrak{g})^{G_{0}}$ and $Z(\mathfrak{U} \mathfrak{g})$ respectively. This is related to Kostant's "separation of variables" theorem; see Equation (2.4), where $\mathbb{H}(\mathfrak{g})$ is precisely the image of the harmonic polynomials in Sym $\mathfrak{g}$ under $\boldsymbol{\lambda}$. (Recall from above that $[\mathbb{H}(\mathfrak{g}): V(\mu)]=\operatorname{dim} V(\mu)_{0}=d_{\mu}$.) Moreover, it is possible to choose all of the basis elements $M_{i}$ (over the center) for $\mathcal{L}^{\mu}$ such that every $M_{i}(V(\mu)$ ) is a subspace of $\operatorname{Sym}^{q_{i}(\mu)} \mathfrak{h}$ for some $q_{i}(\mu) \in \mathbb{Z}_{\geq 0}$. Such elements are called homogeneous. The image of a set of homogeneous generators under $\boldsymbol{\lambda}$ yields a set of $Z(\mathfrak{U} \mathfrak{g})$-module generators for $\mathcal{L}^{\mu}(\mathfrak{U} \mathfrak{g})$.

The zero weight spaces in these modules $\mathcal{L}^{\mu}, \mathcal{L}^{\mu}(\mathfrak{U} \mathfrak{g})$ are of great interest in [PRV2]. For instance, it is easy to check that for all $\nu \in \Lambda^{+}$,

$$
(\mathfrak{U} \mathfrak{g})_{0}(\nu)=\sum_{\mu \in \Lambda^{+}} \sum_{L \in \mathcal{L}^{\mu}(\mathfrak{U} \mathfrak{g})} L\left(V^{+}(\mu ; 0, \nu)\right)
$$

where $V^{+}(\mu ; 0, \nu)$ was defined above Theorem 3.1. (These spaces were used in Theorem 5.1.)

5.3. PRV determinants. We finally define the $P R V$ determinants. (This material is taken from [PRV2, Sections 3,4].) Fix $\mu \in \Lambda^{+}$, and choose a set $\left\{M_{1}, \ldots, M_{d_{\mu}}\right\}$ of homogeneous generators for $\mathcal{L}^{\mu}$. Also choose a basis $\left\{v_{1}, \ldots, v_{d_{\mu}}\right\}$ of $V(\mu)_{0}$. Now define the PRV matrix as:

$$
\mathbf{K}_{\mu}^{\prime}:=\left(\left(\beta^{\Pi}\left(\boldsymbol{\lambda}\left(M_{i}\right) v_{j}\right)\right)\right)_{1 \leq i, j \leq d_{\mu}} \in \mathfrak{g l}_{d_{\mu}}(\operatorname{Sym} \mathfrak{h}), \quad \mathbf{K}_{\mu}^{\prime}(\nu)=\left(\left(\nu\left(\left(\mathbf{K}_{\mu}^{\prime}\right)_{i j}\right)\right)\right) \in \mathfrak{g l}_{d_{\mu}}(\mathbb{C}) .
$$

One of the important results in [PRV2] that has also influenced much future research is the computation of the determinants of these and related matrices. In the following preliminary result, recall by Theorem 3.1 that $\operatorname{dim} V^{+}\left(\mu ; 0,-w_{\circ} \nu\right)=m_{-w_{\circ} \nu, \nu}^{\mu}=\left[V_{\overline{\mathfrak{g}}}(\nu) \otimes V_{\overline{\mathfrak{g}}}(\nu)^{*}: V_{\overline{\mathfrak{g}}}(\mu)\right]$.

Proposition 5.6 ([PRV2]). For all $\mu, \nu \in \Lambda^{+}, \operatorname{rank} \mathbf{K}_{\mu}^{\prime}(\nu)=\operatorname{dim} V^{+}\left(\mu ; 0,-w_{\circ} \nu\right)$. Moreover,

$$
d_{\mu}>0, \lambda \in \mathfrak{h}^{*} \Longrightarrow\left[\widehat{\pi}_{\lambda, 0}: V_{\overline{\mathfrak{g}}}(\mu)\right] \leq \min \left(\operatorname{rank} \mathbf{K}_{-w_{\circ} \mu}^{\prime}(\lambda), \operatorname{rank} \mathbf{K}_{\mu}^{\prime}\left(w_{\circ} * \lambda\right)\right) .
$$

The proposition is proved using the auxiliary lemmas developed in [PRV2, Section 3], and holds for all dominant integral $\mu, \nu$. The authors now propose another matrix which turns out to be nonsingular at all regular points $h \in \mathfrak{h}$. To define this, suppose $M_{i} \in \mathcal{L}^{\mu}, v_{j} \in V(\mu)_{0}$, and $q_{i}(\mu)=\operatorname{deg}\left(M_{i} v_{j}\right) \geq 0$ as above. Now there exists a unique $h_{i j} \in \operatorname{Sym} \mathfrak{h}$ such that $M_{i} v_{j} \equiv h_{i j}$ $\bmod \sum_{\alpha \in R}(\operatorname{Sym} \mathfrak{h}) \mathfrak{g}_{\alpha}$, and moreover, $h_{i j}$ is homogeneous of degree $q_{i}(\mu)$ for all $1 \leq i, j \leq d_{\mu}$. We now introduce the following terminology.

\section{Definition 5.7.}

(1) Define the matrix $\mathbf{K}_{\mu}:=\left(\left(h_{i j}\right)\right)_{1 \leq i, j \leq d_{\mu}}$. 
(2) Fix $\alpha \in R^{+}$, and suppose $\alpha \leftrightarrow h_{\alpha}^{\prime}$ via the Killing form. Now define $h_{\alpha}:=\left(2 / \alpha\left(h_{\alpha}^{\prime}\right)\right) h_{\alpha}^{\prime}$. Let $e_{\alpha}, f_{\alpha}$ span $\mathfrak{g}_{\alpha}, \mathfrak{g}_{-\alpha}$ respectively, and let $m_{j, \mu}(\alpha)$ denote the multiplicity of the eigenvalue $j(j+1)$ for the restriction of $f_{\alpha} e_{\alpha}$ to $V(\mu)_{0}$. Finally, define $m_{\mu}(\alpha)=\sum_{j>0} m_{j, \mu}(\alpha)$.

The following is the main theorem of [PRV2] involving PRV determinants:

Theorem 5.8 ([PRV2]). Fix $\mu \in \Lambda^{+}$. Viewed (via the Killing form) as a polynomial function on $\mathfrak{h}, \operatorname{det} \mathbf{K}_{\mu}$ is nonzero at each regular point $h \in \mathfrak{h}$. In particular, $\operatorname{det} \mathbf{K}_{\mu}$, $\operatorname{det} \mathbf{K}_{\mu}^{\prime}$ are nonzero elements of $P\left(\mathfrak{h}^{*}\right)$ of degree $\sum_{i} q_{i}(\mu)$. Moreover, there exist nonzero constants $c_{\mu}, c_{\mu}^{\prime}$ such that:

$$
\operatorname{det} \mathbf{K}_{\mu}=c_{\mu} \prod_{\alpha \in R^{+}} h_{\alpha}^{m_{\mu}(\alpha)}, \quad \operatorname{det} \mathbf{K}_{\mu}^{\prime}=c_{\mu}^{\prime} \prod_{\alpha \in R^{+}} \prod_{j \geq 1}\left\{h_{\alpha}+\rho\left(h_{\alpha}\right)-1, j\right\}^{m_{j, \mu}(\alpha)},
$$

where for all $a \in \mathfrak{U} \mathfrak{g}$ and $j \in \mathbb{N},\{a, j\}:=(-1)^{j} j ! a(a-1) \ldots(a-j+1)$. (In particular, $\sum_{i=1}^{d_{\mu}} q_{i}(\mu)=\sum_{\alpha \in R^{+}} m_{\mu}(\alpha)$.)

This is a powerful theorem that computes the PRV determinants in a simple manner. It can be used to compute these determinants explicitly for simple $\mathfrak{g}$, with $V(\mu)$ the adjoint representation, for instance. Let us take a simple example: $\mathfrak{g}=\mathfrak{s l}_{2}$. First note that $d_{\mu}>0$ if and only if $\mu \in 2 \mathbb{Z}_{\geq 0} \varpi$. Suppose this holds, and apply Equation (1.1). Then, $f e \cdot v_{0}=(\mu / 2)(\mu / 2+1) v_{0}$, so $m_{j, \mu}(\alpha)=\delta_{j, \mu / 2}$ and $m_{\mu}(\alpha)=1$ for all even $\mu$. Hence

$$
\operatorname{det} \mathbf{K}_{\mu}=c_{\mu} h, \quad \operatorname{det} \mathbf{K}_{\mu}^{\prime}=c_{\mu}^{\prime}\{h, \mu / 2\} \in \mathbb{C}^{\times} \cdot h(h-1) \ldots(h-\mu / 2+1) .
$$

It turns out that this is exactly the Shapovalov determinant for $\mathfrak{s l}_{2}(\mathbb{C})$ (up to a scalar). We now show how these are related to PRV determinants, before moving on to the next section.

5.4. Annihilators of Verma modules. Since they were defined and computed in PRV2], PRV determinants have played a role in the study of annihilators of Verma modules and their simple quotients, in the following manner. In [Du1, Duflo proved the following remarkable result: The annihilator of every Verma module is centrally generated. In other words, for any complex semisimple $\mathfrak{g}$,

$$
\operatorname{Ann}_{\mathfrak{U} \mathfrak{g}} M(\lambda)=\mathfrak{U} \mathfrak{g} \cdot \operatorname{Ann}_{Z(\mathfrak{U} \mathfrak{g})} M(\lambda)=\mathfrak{U} \mathfrak{g} \cdot \operatorname{ker} \chi(\lambda), \quad \forall \lambda \in \mathfrak{h}^{*} .
$$

The proof of this statement requires a nontrivial algebro-geometric argument from [Ko2]. Thus, it cannot be extended to the setting of quantum groups $U_{q}(\mathfrak{g})$, and a new proof was sought. This was provided by Joseph for quantum groups, but it holds in the classical setting as well. In this part, we discuss how PRV determinants play a role in proving Duflo's result.

Given a weight $\mu \in \mathfrak{h}^{*}$, every weight space $M(\mu)_{\mu-\beta}$ of a Verma module has a bilinear form defined on it (where $\beta \in \mathbb{Z}_{\geq 0} \Pi$ ). To see this, first fix a Lie algebra anti-involution $\iota: \mathfrak{g} \rightarrow \mathfrak{g}$ that fixes $\mathfrak{h}$ and sends $e_{i}$ to $f_{i}$ for all $i \in I$. Then $\iota$ sends $\mathfrak{g}_{\alpha}$ to $\mathfrak{g}_{-\alpha}$ for all roots $\alpha$, and also extends to an algebra anti-involution of $\mathfrak{U} \mathfrak{g}$. Now the Shapovalov form is defined as follows:

$$
\operatorname{Sh}\left(b_{1}, b_{2}\right):=\beta^{\Pi}\left(\iota\left(b_{1}\right) \cdot b_{2}\right) \in \operatorname{Sym} \mathfrak{h}, \quad \operatorname{Sh}_{\mu}\left(b_{1} m_{\mu}, b_{2} m_{\mu}\right):=\mu\left(\operatorname{Sh}\left(b_{1}, b_{2}\right)\right), \quad \forall b_{1}, b_{2} \in \mathfrak{U n}^{-},
$$

where $m_{\mu} \in M(\mu)_{\mu}$ generates the Verma module. One shows that the nondegeneracy of this form can be checked on each individual weight space. Thus for all $\nu \in \mathbb{Z}_{\geq 0} \Pi$, let $\operatorname{det} \mathrm{Sh}^{\nu}$ be the determinant of $\mathrm{Sh}$, when restricted to a (fixed) weight space basis of $\left(\mathfrak{U}^{-}\right)_{-\nu}$. The Shapovalov form can now be shown to possess the following properties (see [MP, [Sh] for instance):

Theorem 5.10. For all $\mu \in \mathfrak{h}^{*}, \mathrm{Sh}_{\mu}$ is a symmetric bilinear form on $M(\mu) . \operatorname{Sh}_{\mu}\left(M(\mu)_{\nu}, M(\mu)_{\nu^{\prime}}\right)$ is nonzero only when $\nu=\nu^{\prime} \in \mu-\mathbb{Z}_{\geq 0} \Pi$. Moreover, the radical of $\mathrm{Sh}_{\mu}$ is the unique maximal 
submodule of $M(\mu)$, so it is nondegenerate if and only if $M(\mu) \cong V(\mu)$. Finally, there exists a nonzero constant $c_{\nu}^{\prime \prime}$ such that

$$
\operatorname{det} \mathrm{Sh}^{\nu}=c_{\nu}^{\prime \prime} \prod_{\alpha \in R^{+}} \prod_{j \geq 1}\left(h_{\alpha}+\rho\left(h_{\alpha}\right)-j\right)^{\mathcal{P}(\nu-j \alpha)} .
$$

We note that both the PRV and Shapovalov determinants $\operatorname{det} \mathbf{K}_{\mu}^{\prime}$, $\operatorname{det} \mathrm{Sh}^{\nu}$ are products of linear factors. However, more holds! (The rest of this part is from [FL.)

Theorem 5.11. For all semisimple $\mathfrak{g}$, the set of all linear factors in $\left\{\operatorname{det} \mathbf{K}_{\mu}^{\prime}: \mu \in \Lambda^{+}, d_{\mu}>0\right\}$ and $\left\{\operatorname{det} \mathrm{Sh}^{\nu}: \nu \in \mathbb{Z}_{\geq 0} \Pi\right\}$ coincide. Moreover, given $\lambda \in \mathfrak{h}^{*}$, the annihilator $\operatorname{Ann}_{\mathbb{H}(\mathfrak{g})} V(\lambda)$ is trivial if and only $\lambda\left(\operatorname{det} \mathbf{K}_{\mu}^{\prime}\right)=0$ whenever $d_{\mu}>0$.

Thus, another approach to proving Duflo's "Verma module annihilator theorem" (5.9) is to proceed as follows. This is a program developed by Joseph and his coauthors.

(1) $U:=\mathfrak{U} \mathfrak{g}$ has a large "locally finite subalgebra" $F(U):=\{a \in U: \operatorname{dim}(\operatorname{ad} U) a<\infty\}$.

(2) A "Peter-Weyl" type result holds: $F(U):=\bigoplus_{\lambda \in \Lambda^{+}} \operatorname{End}_{\mathbb{C}} V(\lambda)$. (Note that for $U=\mathfrak{U} \mathfrak{g}$, this is proved for $F(U)=U$ using the perfect pairing between left-invariant differential operators in $\mathfrak{U} \mathfrak{g}$ and regular functions on the simply connected Lie group $G$ for which $\mathfrak{g}=\operatorname{Lie}(G)$, together with the usual Peter-Weyl Theorem for regular functions on $G$.) Under this identification, the lifts of the identity elements in the various summands are a basis $\left\{z_{\lambda}: \lambda \in \Lambda^{+}\right\}$of the center; moreover, $Z(U)$ is a polynomial algebra in the generators $\left\{z_{\varpi_{i}}: i \in I\right\}$.

(3) There exists an ad $U$-stable submodule $\mathbb{H}$ of $F(U)$ such that the multiplication map $: \mathbb{H} \otimes Z(U) \rightarrow F(U)$ is an isomorphism of ad $U$-modules 6 Here, ad is the standard adjoint action of the Hopf algebra $U$ on itself.

(4) Now define the PRV determinants using the above facts, and the Shapovalov determinants using the anti-involution $\iota$ and the Harish-Chandra projection $\beta^{\Pi}$. Then calculate both sets of determinants and verify their properties as in the above results.

(5) Now use the PRV and Shapovalov determinants for any simple submodule of a Verma module $M(\lambda)$, which is itself a Verma module with the same central character $\chi(\lambda)$. Some more work now shows Duflo's result.

The important point is that this approach works not only for $U=\mathfrak{U} \mathfrak{g}$, but also for $U=U_{q}(\mathfrak{g})$. (Unlike $\mathfrak{U} \mathfrak{g}, F(U) \neq U$ in the quantum case.) Thus, Joseph and Letzter proved the quantum "separation of variables" theorem, and defined and computed the related PRV determinants. See [JL1, JL2]; also see [FL] for a historical exposition of this program.

Joseph and others have since extended this approach to affine Lie algebras. Similarly, Gorelik and Lanzmann GL have also carried out this program for reductive super Lie algebras. They found that the PRV determinants contained some "extra factors" compared to the Shapovalov determinants, and their zeroes are precisely the weights for which the corresponding Verma module annihilators are not centrally generated. Thus, the PRV and Shapovalov determinants (or more precisely, their common zeroes) turn out to yield, in various settings, both an approach to proving Duflo's Theorem (5.9), as well as the set of Verma modules for which it holds.

5.5. KPRV determinants. We end this section with a remark. Kostant described certain analogues of the PRV determinants in [Ko3] involving parabolic subalgebras of $\mathfrak{g}$; these analogues had applications related to the irreducibility of principal series representations. Joseph termed these the KPRV determinants, and together with Letzter and with Todoric, has defined such

\footnotetext{
${ }^{6}$ If $U=\mathfrak{U} \mathfrak{g}$, then $F(U)=U$, and this result is precisely Equation (2.4). One can also use this and Example 3.2 to prove the Peter-Weyl type result mentioned above, by counting (countably infinite) multiplicities.
} 
notions for (quantum) semisimple and affine Lie algebras. (See [Jo3, JL3, JLT, JT] for more on this, including applications to annihilators of Verma modules.) Thus, the PRV determinants and their generalizations continue to be a useful and popular subject of research in several different settings in representation theory.

\section{Representations of Class ZERo}

In [PRV2, Section 4], the authors apply the theory previously developed to carry out a deeper study of a special sub-family of irreducible admissible $(\mathfrak{g} \times \mathfrak{g}, \overline{\mathfrak{g}})$-modules: the ones of "class zero". Here is a brief discussion of these modules and related results.

Definition 6.1. An irreducible $\overline{\mathfrak{g}}$-admissible $G$-representation $V$ is said to be of class zero if $\left[V: V_{\overline{\mathfrak{g}}}(0)\right]>0$.

(Note that these are usually referred to as "class one" representations in the literature - i.e., irreducible admissible $(G, K)$-modules which have a $K$-invariant vector. In other words, "class one" refers to a $K$-eigenvector with simultaneous eigenvalue 1 , while "class zero" refers to a $\overline{\mathfrak{g}}$ eigenvector with simultaneous eigenvalue 0 .) The first result says that every irreducible HarishChandra module of class zero is determined by its central character; in fact, it is of the form $\widehat{\pi}_{\lambda, 0}$ for some $\lambda \in \mathfrak{h}^{*}$. Thus, we obtain deeper insights into the classification of such modules.(See Equation (2.8) and Theorem 4.9.)

Theorem 6.2 ([PRV2]). The set of infinitesimal equivalence classes of class zero irreducible representations $V$ is in bijection with the twisted Weyl group orbits in $\mathfrak{h}^{*}$. More precisely, every such $V$ is uniquely determined by its infinitesimal character $\chi_{V}$ restricted to $Z(\mathfrak{U} \mathfrak{g})_{(1)}$. Moreover, given $\chi_{V}: Z(\mathfrak{U}(\mathfrak{g} \times \mathfrak{g})) \rightarrow \mathbb{C}$, there exists $\lambda \in \mathfrak{h}^{*}$ such that

$$
\chi_{V}=\chi(\lambda,-\lambda-2 \rho), \quad V \cong \widehat{\pi}_{\lambda, 0} \cong \widehat{\pi}_{w * \lambda, 0} \forall w \in W .
$$

In particular $\left[V: V_{\overline{\mathfrak{g}}}(0)\right]=1$ and 0 is the minimal type of $V$.

Example 6.3. If $\lambda \in \Lambda^{+}$, then $\chi_{\widehat{\pi}_{\lambda, 0}}=\chi\left(\lambda, w_{\circ} *(-\lambda-2 \rho)\right)=\chi\left(\lambda,-w_{\circ} \lambda\right)=\chi_{V(\lambda) \otimes V(\lambda)^{*}}$. By Theorem 3.5, the minimal type of $V(\lambda) \otimes V(\lambda)^{*}$ is $\lambda+w_{\circ}\left(-w_{\circ} \lambda\right)=0$ as well. Therefore $\widehat{\pi}_{\lambda, 0} \cong V(\lambda) \otimes V(\lambda)^{*}$ by the above result. Moreover, every finite-dimensional $\widehat{\pi}_{\lambda, 0}$ is of this kind, by Corollary 4.6 .

We now take a closer look at the multiplicities. When is $\left[\widehat{\pi}_{\lambda, 0}: V_{\overline{\mathfrak{g}}}(\mu)\right]=\operatorname{dim} V_{\overline{\mathfrak{g}}}(\mu)_{0}$ ? (That it is at most $\operatorname{dim} V_{\overline{\mathfrak{g}}}(\mu)_{0}$ follows from Theorem 4.1.) More generally, is it possible to compute the multiplicities for class zero modules? Once again, the authors were able to achieve this goal in [PRV2]: the multiplicity equals the rank of a related matrix, which is defined similar to $\mathbf{K}_{\mu}^{\prime}$ above. Namely, given $\mu \in \Lambda^{+}$such that $d_{\mu}>0$, choose sets of homogeneous generators $\left\{M_{1}, \ldots, M_{d_{\mu}}\right\}$ and $\left\{M_{1}^{*}, \ldots, M_{d_{\mu}}^{*}\right\}$ for the free $(\operatorname{Sym} \mathfrak{g})^{G_{0}}$-modules $\mathcal{L}^{\mu}$ and $\mathcal{L}^{-w_{\circ} \mu}$ respectively. Now choose dual bases $\left\{v_{k}\right\}$ and $\left\{v_{k}^{*}\right\}$ for $V(\mu), V\left(-w_{\circ} \mu\right) \cong V(\mu)^{*}$ respectively. The span of

$$
z_{i j}:=\sum_{k}\left(\boldsymbol{\lambda}\left(M_{i}^{*}\right) v_{k}^{*}\right)\left(\boldsymbol{\lambda}\left(M_{j}\right) v_{k}\right)
$$

then depends only on $M_{i}$ and $M_{j}$. One can now compute the sought-for multiplicities.

Theorem 6.4 ([PRV2] $)$. For all $\mu \in \Lambda^{+}$and $1 \leq i, j \leq d_{\mu}, z_{i j} \in Z(\mathfrak{U} \mathfrak{g})$. Now given a central character $\chi=\chi(\lambda)$ of $Z(\mathfrak{U} \mathfrak{g})$ (where $\left.\lambda \in \mathfrak{h}^{*}\right),\left[\widehat{\pi}_{\lambda, 0}: V_{\overline{\mathfrak{g}}}(\mu)\right]=\mathbf{1}_{d_{\mu}>0} \cdot \operatorname{rank}\left(\left(\chi(\lambda)\left(z_{i j}\right)\right)\right)$.

The next result discusses the case when the multiplicities all attain their upper bounds. This turns out to be an important question from the point of view of the irreducibility of the induced representations $\pi_{\xi, \nu}$ discussed earlier; see also $[\mathrm{Bru}$. The following result completely answers this question. (See Du2] for more results along these lines.) 
Theorem 6.5 ([R2]). Given $\lambda \in \mathfrak{h}^{*}$, the following are equivalent:

(1) $\widehat{\pi}_{\lambda, 0}$ is complete, i.e., $\left[\widehat{\pi}_{\lambda, 0}: V_{\overline{\mathfrak{g}}}(\mu)\right]=\operatorname{dim} V_{\overline{\mathfrak{g}}}(\mu)_{0}$ whenever $d_{\mu}>0$ for $\mu \in \Lambda^{+}$.

(2) $\pi_{2(\lambda+\rho), 0}$ is irreducible.

(3) The matrices $\mathbf{K}_{-w_{\circ} \mu}^{\prime}(\lambda)$ and $\mathbf{K}_{\mu}^{\prime}\left(w_{\circ} * \lambda\right)$ are both invertible whenever $d_{\mu}>0$ for $\mu \in \Lambda^{+}$.

(4) For all roots $\alpha \in R^{+}, \frac{1}{2} \xi\left(h_{\alpha}\right)=(\lambda+\rho)\left(h_{\alpha}\right) \notin \mathbb{Z} \backslash\{0\}$.

This result now holds when $\xi$ attains purely imaginary values on $\mathfrak{h}_{0}$ (i.e., $\xi \in \mathfrak{R} \backslash\{0\}$, in the notation of Theorem 4.1). This shows the irreducibility of a class of unitary representations that was studied previously in the complex semisimple case:

Theorem 6.6 ([PRV2]). The unitary G-representations of the principal nondegenerate series (of Gelfand and Naimark [GN]) that contain a nonzero $K$-invariant vector, are all irreducible.

\section{Conclusion: The Classification of irreducible Harish-Chandra modules}

As discussed in previous sections, the paper PRV2 has led to much research in several different directions in representation theory. In this final section, we return to its original motivations. As is evident from the paper, as well as from much of the contemporary literature, the fundamental and profound work of Harish-Chandra on semisimple (real) Lie groups has had an enormous influence on the field of representation theory. From the objects studied to the methods employed in [PRV2], the authors have time and again used contributions of HarishChandra to the subject.

We now mention some of the subsequent developments in the program started by HarishChandra, of studying $K$-admissible $G$-representations. For instance, various results from Har2, Har3, PRV2] were subsequently generalized by Lepowsky in [Le]. Moreover, in [Zh1, Zh2], Zhelobenko classified irreducible admissible representations of complex semisimple Lie groups, by showing that they always arise as distinguished quotients of certain principal series representations. This classification is the subject of this section.

Before moving on to these classification results, we remark that this program was extended by Langlands in [La], to the original setting of real semisimple Lie groups $G_{\mathbb{R}}$, where Harish-Chandra had introduced and studied admissible representations. The Langlands classification describes how irreducible admissible representations are quotients of "generalized principal series", which are induced from tempered representations on parabolic subgroups of $G_{\mathbb{R}}$. The work of Langlands and Harish-Chandra on tempered representations was refined by Knapp and Zuckerman [KZ]; thus, one now has an explicit parametrization of the irreducible admissible representations of the groups $G_{\mathbb{R}}$. (See also [BB], which studies more general irreducible representations than just the admissible ones.) As this suggests, the legacy of Harish-Chandra is vast and rich, and lives on in these works and in the subsequent research which it has inspired.

7.1. The set of irreducible objects. We now discuss the classification of all irreducible objects of $\mathcal{C}(\mathfrak{g} \times \mathfrak{g}, \overline{\mathfrak{g}})$. There are two parts to this discussion: first, to determine a representative set of simple objects that covers all isomorphism classes; and second, to determine the equivalences among the objects in this set. In what follows, the final results will be stated as they appear in Duflo's notes Du2] on the subject.

It turns out that the category $\mathcal{C}(\mathfrak{g} \times \mathfrak{g}, \overline{\mathfrak{g}})$ is equivalent to a subcategory of the BGG Category $\mathcal{O}$. In particular, Harish-Chandra modules have certain properties in common with Verma modules. For instance, all objects of this category have finite length, all simple objects have a corresponding central character, and for a given central character, the simple objects are indexed by the Weyl group. More precisely, Beilinson and Bernstein have classified all irreducible $(\mathfrak{g} \times \mathfrak{g}, \overline{\mathfrak{g}})$-modules with a fixed infinitesimal character. Here is a special case of their results. 
Theorem $7.1([\mathrm{BB}])$. Given $\lambda, \mu \in \Lambda^{+}$, the set of isomorphism classes of irreducible admissible $(\mathfrak{g} \times \mathfrak{g}, \overline{\mathfrak{g}})$-modules with infinitesimal character $\chi=\chi(\lambda, \mu)$ is in bijection with $W_{\lambda} \backslash W / W_{\mu}$.

For instance, if $\lambda, \mu$ are both regular, then there are exactly $|W|$ isomorphism classes, while there is a single class if $\lambda$ or $\mu$ is zero.

Now recall Theorem 6.2, which says that all irreducible admissible class zero modules are of the form $\widehat{\pi}_{\lambda, 0}$ for some $\lambda \in \mathfrak{h}^{*}$. One can similarly ask: is every irreducible admissible $(\mathfrak{g} \times \mathfrak{g}, \overline{\mathfrak{g}})$ module of the form $\widehat{\pi}_{\lambda, \nu}$ for some $(\lambda, \nu) \in \mathfrak{h}^{*} \times \Lambda$ ? The answer turns out to be positive.

Theorem 7.2. Suppose $V$ is an irreducible object of $\mathcal{C}(\mathfrak{g} \times \mathfrak{g}, \overline{\mathfrak{g}})$. Then there exist $\lambda \in \mathfrak{h}^{*}$ and $\nu \in \Lambda$ such that $V \cong \widehat{\pi}_{\lambda, \nu} \cong \widehat{\pi}_{w * \lambda, w \nu}$ for all $w \in W$. In particular, $V$ has minimal type $\bar{\nu}$ and infinitesimal character $\chi(\lambda, \nu-\lambda-2 \rho)$.

7.2. The objects in a given isomorphism class. The other aspect of classification is to identify the isomorphism classes. In light of the above result, the task is to identify when $\widehat{\pi}_{\lambda, \nu} \cong \widehat{\pi}_{\lambda^{\prime}, \nu^{\prime}}$. In light of Corollary 4.3, one may assume that $\nu=\nu^{\prime} \in \Lambda^{+}$. Moreover, in light of Corollaries 4.3 and 5.3 for general $\mathfrak{g}$, and Equation (5.5) for $\mathfrak{s l}_{2}(\mathbb{C})$, it is easy to guess the general result. This is further reinforced by the fact that if $\nu, \nu^{\prime} \in \Lambda$, and $\xi \in \mathfrak{R}_{\nu}, \xi^{\prime} \in \mathfrak{R}_{\nu^{\prime}}$ (notation as in Theorem 4.1), then $\pi_{\xi, \nu}$ is irreducible, hence isomorphic to $\widehat{\pi}_{\lambda, \nu}$ (and similarly for $\pi_{\xi^{\prime}, \nu^{\prime}}$ ). Now as observed in PRV2,

$$
\begin{gathered}
\widehat{\pi}_{\lambda, \nu} \cong \widehat{\pi}_{\lambda^{\prime}, \nu^{\prime}} \Longleftrightarrow \pi_{\xi, \nu} \cong \pi_{\xi^{\prime}, \nu^{\prime}} \Longleftrightarrow \Theta_{\xi, \nu}=\Theta_{\xi^{\prime}, \nu^{\prime}} \\
\Longleftrightarrow \quad \exists w \in W:\left(\xi^{\prime}, \nu^{\prime}\right)=(w \xi, w \nu) \Longleftrightarrow \exists w \in W:\left(\lambda^{\prime}, \nu^{\prime}\right)=(w * \lambda, w \nu) .
\end{gathered}
$$

It should not come as a surprise now, that the obvious claim turns out to be correct:

Theorem 7.3. Given $(\lambda, \nu),\left(\lambda^{\prime}, \nu^{\prime}\right) \in \mathfrak{h}^{*} \times \Lambda$,

$$
\widehat{\pi}_{\lambda, \nu} \cong \widehat{\pi}_{\lambda^{\prime}, \nu^{\prime}} \Longleftrightarrow \exists w \in W:\left(\lambda^{\prime}, \nu^{\prime}\right)=(w * \lambda, w \nu) .
$$

7.3. Concluding remarks. We end with a couple of (incomplete) calculations regarding the above analysis, involving central characters.

(1) It is natural to ask if the central character associated to an irreducible admissible module $V$, determines its minimal type. Thus, given that $\chi_{V}=\chi\left(\mu_{1}, \mu_{2}\right)$, how does one determine the minimal type of $V$ ?

It is clear that if $V \cong \widehat{\pi}_{\lambda, \nu}$ (from above results), then

$$
\mu_{1}=w_{1} * \lambda, \quad \mu_{2}=w_{2} *(\nu-\lambda-2 \rho)=w_{2} \nu-w_{2} * \lambda-2 \rho,
$$

for some $w_{1}, w_{2} \in W$. Now note that

$$
\mu_{1}+w_{1} w_{2}^{-1} * \mu_{2}=w_{1} * \lambda+w_{1} \nu-w_{1} * \lambda-2 \rho=w_{1} \nu-2 \rho .
$$

Hence $\nu_{w}:=2 \rho+\mu_{1}+w * \mu_{2} \in \Lambda$ for some $w \in W$; moreover, for every such $w, \overline{\nu_{w}}$ is a candidate for the minimal type, by these calculations. Thus, if $w$ is not uniquely identified from above, then neither is $\bar{\nu}$.

(2) Similarly, given $(\lambda, \nu),\left(\lambda^{\prime}, \nu^{\prime}\right) \in \mathfrak{h}^{*} \times \Lambda$, a necessary condition for $\widehat{\pi}_{\lambda, \nu}$ to be isomorphic to $\widehat{\pi}_{\lambda^{\prime}, \nu^{\prime}}$ is that their central characters and minimal types coincide. It is natural to ask if this data is also sufficient to determine the isomorphism type.

Clearly, in order to have the same minimal type, Corollary 4.3 implies that $\nu^{\prime} \in W \nu$. Say $\nu^{\prime}=w_{1} \nu$. Now since the infinitesimal characters coincide, Theorem 4.9 implies:

$$
\lambda^{\prime}=w_{2} * \lambda, \quad \nu^{\prime}-\lambda^{\prime}-2 \rho=w *(\nu-\lambda-2 \rho)=w \nu-w * \lambda-2 \rho .
$$

Using these equations translates to the following condition:

$$
w_{1} \nu-w_{2} * \lambda=w \nu-w * \lambda
$$


and this data may not have the unique solution: $w_{1}=w_{2}=w$.

The reason for this discrepancy is Equation (2.8): the representation $\widehat{\pi}_{\lambda, \nu}$ carries the same data as its minimal type and the action of $\Omega$ on it. The above data only accounts for the minimal type and the action of the proper subset $Z(\mathfrak{U}(\mathfrak{g} \times \mathfrak{g})) \subsetneq \Omega$. For instance, $Z(\mathfrak{U} \overline{\mathfrak{g}})$ is not accounted for.

To conclude, we have tried to explain the flavour of some of the results in [PRV2], as well as their connection to, and impact on, subsequent research in a wide variety of directions in the field. From the multiplicity problem and obtaining components in tensor products of finite-dimensional modules, to PRV determinants and annihilators of Verma modules, to the classification of all irreducible admissible modules as in Harish-Chandra's grand program on semisimple Lie groups - the work [PRV2] has contributed to, and inspired much subsequent research in, many aspects of representation theory. The list of results and connections mentioned in this article is by no means complete, but we hope that it suffices to convince the reader of the importance and influence of this work in representation theory.

Acknowledgments. I would first like to thank Professors C.S. Rajan and Rajendra Bhatia for kindly inviting me to write this article. Next, I greatly thank Professor Shrawan Kumar for his generous help in pointing out several references and follow-up results in the literature. I would also like to thank very much Professor Dipendra Prasad, for his time and patience in answering several of my questions, as well as Professor Vyjayanthi Chari for useful references and discussions. An excellent historical account of the writing of [PRV2] can be found in Professor V.S. Varadarajan's reminiscences [Va], and this was of great help in the writing of the present work as well.

\section{REFERENCES}

[BB] A.A. Beilinson and J.N. Bernstein, Localisation de g-modules, C. R. Acad. Sci. Paris, Ser. 1292 (1981), $15-18$.

[BZ] A. Berenstein and A. Zelevinsky, Tensor product multiplicities, canonical bases and totally positive varieties, Inventiones Mathematicae 143 (2001), 77-128.

[BGG] J. Bernstein, I.M. Gelfand, and S.I. Gelfand, A category of $\mathfrak{g}$ modules, Functional Analysis and Applications 10 (1976), 87-92.

[Br] R. Brauer, Sur la multiplication des caractéristiques des groups continus et semi-simples, C. R. Acad. Sci. Paris 204 (1937), 1784-1786.

[Bru] F. Bruhat, Sur les réprésentations induites des groupes de Lie, Bull. Soc. Math. France 84 (1956), $97-205$.

[CG1] V. Chari and J. Greenstein, Current algebras, highest weight categories and quivers, Advances in Mathematics 216 (2007), no. 2, 811-840.

[CG2] - Minimal affinizations as projective objects, Journal of Geometry and Physics 61 (2011), no. 3, 594-609.

[CKR] V. Chari, A. Khare, and T.B. Ridenour, Faces of polytopes and Koszul algebras, Journal of Pure and Applied Algebra 216 (2012), no. 7, 1611-1625.

[CP] V. Chari and A. Pressley, A new family of irreducible, integrable modules for affine Lie algebras, Mathematische Annalen 277 (1987), no. 3, 543-562.

[DR] I. Dimitrov and M. Roth, Geometric realization of PRV components and the Littlewood-Richardson cone, Contemporary Mathematics 490: Symmetry in Mathematics and Physics, D. Babbitt, V. Chari, and R. Fioresi, Eds. (2009), 83-95.

[Du1] M. Duflo, Construction of primitive ideals in an enveloping algebra, Lie groups and their representations (1971 János Bolyai Math. Soc. Summer School in Mathematics, Budapest), I.M. Gelfand, Ed. (1975), 77-93.

[Du2] — Représentations irréductibles des groupes semi-simples complexes, Springer Lecture Notes in Mathematics 497 (1975), 26-88.

[Du3] - Sur la classification des idéaux primitifs dans l'algèbre enveloppante d'une algèbre de Lie semisemisimple, Annals of Mathematics 105 (1977), 107-120. 
[FL] D.R. Farkas and G. Letzter, Quantized representation theory following Joseph, Progress in Mathematics 243, Part I: Studies in Lie Theory (2006), 9-17.

[GN] I.M. Gelfand and M.A. Naimark, Unitary representations of the classical groups, Trudy Mat. Inst. Steklov 36, Moscow-Leningrad, 1950.

[GL] M. Gorelik and E. Lanzmann, The annihilation theorem for Lie superalgebra osp $(1,2 \ell)$, Inventiones Mathematicae 137 (1999), 651-680.

[Hal] B.C. Hall, Lie groups, Lie algebras, and representations: an elementary introduction, Graduate Texts in Mathematics, no. 222, Springer-Verlag, Berlin-New York, 2004.

[Har1] Harish-Chandra, On some applications of the universal enveloping algebra of a semi-simple Lie algebra, Trans. Amer. Math. Soc. 70 (1951), 28-96.

[Har2] , Representations of a semi-simple Lie group on a Banach space: I, Trans. Amer. Math. Soc. 75 (1953), 185-243.

[Har3] , Representations of semi-simple Lie groups: II, Trans. Amer. Math. Soc. 76 (1954), 26-65.

[Har4] , The Plancherel formula for complex semi-simple Lie groups, Trans. Amer. Math. Soc. 76 (1954), $485-528$.

[Hay] M. Hayashi, The moduli space of SU(3)-flat connections and the fusion rules, Proc. Amer. Math. Soc. 127 (1999), 1545-1555.

[Hu1] J.E. Humphreys, Introduction to Lie algebras and representation theory, Graduate Texts in Mathematics, no. 9, Springer-Verlag, Berlin-New York, 1972.

[Hu2] - Representations of semisimple Lie algebras in the BGG Category $\mathcal{O}$, Graduate Studies in Mathematics 94, American Mathematical Society, Providence, RI, 2008.

[Jo1] A. Joseph, Quantum groups and their primitive ideals, Ergeb. Math. Grenzgeb. (3) 29, Springer, Berlin, 1995.

[Jo2] _ A completion of the quantized enveloping algebra of a Kac-Moody algebra, Journal of Algebra 214 (1999), no. 1, 235-275.

[Jo3] - On the Kostant-Parthasarathy-Ranga Rao-Varadarajan determinants, I. Injectivity and multiplicities, Journal of Algebra 241 (2001), 27-45.

[JL1] A. Joseph and G. Letzter, Separation of variables for quantized enveloping algebras, American Journal of Mathematics 116 (1994), 127-177.

[JL2] U Verma modules annihilators for quantized enveloping algebras, Ann. Ecole Norm. Sup. 28 (1995), $493-526$.

[JL3] On the Kostant-Parthasarathy-Ranga Rao-Varadarajan determinants, II. Construction of the KPRV determinants, Journal of Algebra 241 (2001), 46-66.

[JLT] A. Joseph, G. Letzter, and D. Todoric, On the Kostant-Parthasarathy-Ranga Rao-Varadarajan determinants, III. Computation of the KPRV determinants, Journal of Algebra 241 (2001), 67-88.

[JT] A. Joseph and D. Todoric, On the quantum KPRV determinants for semisimple and affine Lie algebras, Algebras and Representation Theory 5 (2002), 57-99.

[Ka] M. Kashiwara, On crystal bases, Canadian Math. Soc. Conf. Proc. 16 (1995), 155-197.

[KLV] D. Kazhdan, M. Larsen, and Y. Varshavsky, The Tannakian Formalism and the Langlands Conjectures, preprint, math.NT/1006.3864

[Kh] A. Khare, Axiomatic framework for the BGG Category O , preprint, math.RT/0811.2080.

[Kl] A.U. Klimyk, On the multiplicities of weights of representations and the multiplicities of representations of semisimple Lie algebras, Dokl. Acad. Nauk SSSR 177 (1967), 1001-1004.

[KZ] A.W. Knapp and G. Zuckerman, Classification of irreducible tempered representations of semisimple groups, Annals of Mathematics 116 (1982), 389-455.

[Ko1] B. Kostant, A formula for the multiplicity of a weight, Trans. Amer. Math. Soc. 93 (1959), 53-73.

[Ko2] Lie group representations on polynomial rings, American Journal of Mathematics 85 (1963), no. 3, 327-404.

[Ko3] - On the existence and irreducibility of certain series of representations, Lie groups and their representations (1971 János Bolyai Math. Soc. Summer School in Mathematics, Budapest), I.M. Gelfand, Ed. (1975), 231-331.

[Ko4] Clifford algebra analogue of the Hopf-Koszul-Samelson Theorem, the $\rho$-decomposition $C(\mathfrak{g})=$ End $V_{\rho} \otimes C(P)$, and the $\mathfrak{g}$-module structure of $\wedge \mathfrak{g}$, Advances in Mathematics 125 (1997), 275-350.

[Ku1] S. Kumar, Proof of the Parthasarathy-Ranga Rao-Varadarajan conjecture, Inventiones Mathematicae 93 (1988), 117-130. 
[Ku2] _ Existence of certain components in the tensor product of two integrable highest weight modules for Kac-Moody algebras, Advanced series in Mathematical Physics 7: Infinite dimensional Lie algebras and groups, V.G. Kac, Ed. (1989), 25-38.

[Ku3] _ A refinement of the PRV conjecture, Inventiones Mathematicae 97 (1989), 305-311.

[Ku4] Proof of Wahl's conjecture on surjectivity of the Gaussian map for flag varieties, American Journal of Mathematics 114 (1992), 1201-1220.

[Ku5] - Tensor Product Decomposition, Proceedings of the International Congress of Mathematicians (2010).

[La] R.P. Langlands, On the classification of irreducible representations of real algebraic groups, Mathematical Surveys and Monographs 31: Representation theory and harmonic analysis on semisimple Lie groups, P.J. Sally and D.A. Vogan, Eds. (1989), 101-170.

[Le] J. Lepowsky, Algebraic results on representations of semisimple Lie groups, Trans. Amer. Math. Soc. 176 (1973), 1-44.

[LMC] J. Lepowsky and G.W. McCollum, On the determination of irreducible modules by restriction to a subalgebra, Trans. Amer. Math. Soc. 176 (1973), 45-57.

[Li] P. Littelmann, A Littlewood-Richardson rule for symmetrizable Kac-Moody algebras, Inventiones Mathematicae 116 (1994), 329-346.

[Lu] G. Lusztig, Canonical bases arising from quantized enveloping algebras II, Prog. Theor. Phys. 102 (1990), $175-201$.

[Ma1] O. Mathieu, Construction d'un groupe de Kac-Moody et applications, Compositio Mathematica 69 (1989), no. $1,37-60$.

[Ma2] Classification of Harish-Chandra modules over the Virasoro Lie algebra, Inventiones Mathematicae 107 (1992), 225-234.

[Ma3] Classification of irreducible weight modules, Annales de l'institut Fourier 50 (2000), no. 2, 537592.

[MPR1] P.L. Montagard, B. Pasquier, and N. Ressayre, Two generalizations of the PRV conjecture, Composition Mathematica 147 (2011), no. 4, 1321-1336.

[MPR2] — Generalizations of the PRV conjecture, II, preprint, math.RT/1110.4621

[MP] R.V. Moody and A. Pianzola, Lie algebras with triangular decompositions, Canadian Mathematical Society Series of Monographs and Advanced Texts, Wiley Interscience, New York-Toronto, 1995.

[PY] D.I. Panyushev and O.S. Yakimova, The PRV-formula for tensor product decompositions and its applications, Functional Analysis and Applications 42 (2008), no. 1, 45-52.

[PRV1] K.R. Parthasarathy, R. Ranga Rao, and V.S. Varadarajan, Representations of complex semisimple Lie groups and Lie algebras, Bull. Amer. Math. Soc. 72 (1966), 522-525.

[PRV2] _ Representations of complex semisimple Lie groups and Lie algebras, Annals of Mathematics $\mathbf{8 5}$ (1967), 383-429.

[Po] P. Polo, Variétés de Schubert et excellentes filtrations, Astérisque (Orbites unipotentes et représentations) 173-174 (1989), 281-311.

[Ra] K.N. Rajeswari, Standard monomial theoretic proof of PRV conjecture, Communications in Algebra 19 (1991), 347-425.

[Sh] N.N. Shapovalov, On a bilinear form on the universal enveloping algebra of a complex semisimple Lie algebra, Functional Analysis and Its Applications 6 (1972), 307-312.

[St] R. Steinberg, A general Clebsch-Gordan Theorem, Bull. Amer. Math. Soc. 67 (1961), 406-407.

[Va] V.S. Varadarajan, Some mathematical reminiscences, Methods and Applications of Analysis 9 (2002), no. 3, v-xviii.

[Ve] D.N. Verma, Structure of certain induced representations of complex semisimple Lie algebras, Bull. Amer. Math. Soc. 74 (1968), no. 1, 160-166.

[Vo] D.A. Vogan Jr., The algebraic structure of the representation of semisimple Lie groups. I, Annals of Mathematics 109 (1979), no. 1, 1-60.

[YZ] C.A.S. Young and R. Zegers, Dorey's rule and the q-characters of simply-laced quantum affine algebras, Commun. Math. Phys. 302 (2011), 789-813.

[Zh1] D.P. Zhelobenko, The analysis of irreducibility in the class of elementary representations of a complex semisimple Lie group, Math. USSR Izv. 2 (1968), no. 1, 105-128.

[Zh2] Harmonic analysis on complex semisimple Lie groups (Russian), Mir, Moscow, 1974. 